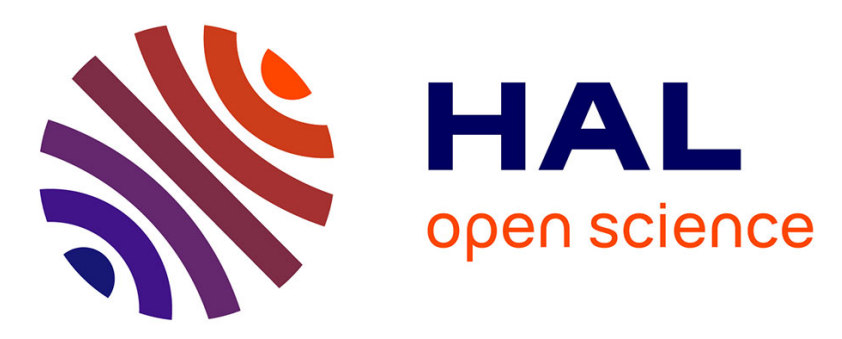

\title{
Three-dimensional continuation study of convection in a tilted rectangular enclosure.
}

\author{
Juan Felipe Torres, Daniel Henry, Atsuki Komiya, Shigenao Maruyama, \\ Hamda Ben Hadid
}

\section{- To cite this version: \\ Juan Felipe Torres, Daniel Henry, Atsuki Komiya, Shigenao Maruyama, Hamda Ben Hadid. Three- dimensional continuation study of convection in a tilted rectangular enclosure.. Physical Review E: Statistical, Nonlinear, and Soft Matter Physics, 2013, 88, pp.043015. 10.1103/PhysRevE.88.043015 . hal-00931500}

\section{HAL Id: hal-00931500 https://hal.science/hal-00931500}

Submitted on 11 Apr 2016

HAL is a multi-disciplinary open access archive for the deposit and dissemination of scientific research documents, whether they are published or not. The documents may come from teaching and research institutions in France or abroad, or from public or private research centers.
L'archive ouverte pluridisciplinaire HAL, est destinée au dépôt et à la diffusion de documents scientifiques de niveau recherche, publiés ou non, émanant des établissements d'enseignement et de recherche français ou étrangers, des laboratoires publics ou privés. 


\title{
Three-dimensional continuation study of convection in a tilted rectangular enclosure
}

\author{
J. F. Torres, ${ }^{1,2}$ D. Henry, ${ }^{1}$ A. Komiya, ${ }^{3}$ S. Maruyama, ${ }^{3}$ and H. Ben Hadid ${ }^{1}$ \\ ${ }^{1}$ Laboratoire de Mécanique des Fluides et d'Acoustique, CNRS/Université de Lyon, Ecole Centrale de Lyon/Université Lyon 1/INSA de Lyon, \\ ECL, 36 avenue Guy de Collongue, 69134 Ecully Cedex, France \\ ${ }^{2}$ Graduate School of Engineering, Tohoku University, 6-6-04, Aramaki Aza Aoba Aoba-ku, Sendai, Miyagi 980-8579, Japan \\ ${ }^{3}$ Institute of Fluid Science, Tohoku University, 2-1-1, Katahira, Aoba-ku, Sendai, Miyagi 980-8577, Japan
}

(Received 25 June 2013; revised manuscript received 20 September 2013; published 30 October 2013)

\begin{abstract}
A continuation method developed from a three-dimensional spectral finite element code is used to study natural convection in a tilted rectangular cavity. The cavity has its length equal to two times the side of its square cross section and it contains a fluid with a Prandtl number $\operatorname{Pr}=1$. A detailed bifurcation diagram is first obtained in the case without inclination in order to get the sequence of the different branches of solutions and determine the stable solutions. The focus is then put on the stable solutions in the inclined cavity, when the tilt occurs around its longest axis. The subtle changes induced by the tilt on the convective system are clarified. Three different stable solutions are obtained: the longitudinal roll $L-$ solution (with the same sense of rotation as the inclination angle), which develops smoothly from zero Rayleigh number on the leading branch; the longitudinal roll $L+$ solution (with a sense of rotation opposite to the inclination angle), which is on a disconnected branch and is stabilized beyond a secondary bifurcation point; the oblique roll $O \pm$ solutions (corresponding to transverse roll solutions perturbed by the longitudinal flow induced by the tilt), which quickly appear beyond saddle-node points on new disconnected branches. The domain of existence of these stable solutions is eventually obtained and described in the Rayleigh number-inclination parameter space. Finally, the Nusselt number is determined as a function of the inclination at a constant Rayleigh number for the different stable solutions. The Nusselt number is maximum at an inclination of $49.55^{\circ}$ for the leading longitudinal roll $L-$ solution.
\end{abstract}

DOI: 10.1103/PhysRevE.88.043015

PACS number(s): 47.20.Bp, 47.20.Ky, 47.11.-j

\section{INTRODUCTION}

Since the pioneering studies of Bénard [1] and Rayleigh [2], the Rayleigh-Bénard convection has been extensively studied. The first interest for these studies was to analyze pattern formation, which was an intriguing phenomenon. This problem, involving both fluid mechanics and heat transfer, is also of great interest in practical applications, as for example crystal growth (see the references in Lappa [3]) and thermal convection in the earth's mantle [4]. The first studies rather concerned infinitely extended layers for which analytical derivations could be performed, but studies in confined enclosures have also developed in connection with practical applications and with the progress in numerical computing [5]. Rayleigh-Bénard convection is an interesting problem for the dynamics of the flows which develop from the motionless conductive solution [6]. A critical Rayleigh number $\mathrm{Ra}_{c}$ has to be reached for the onset of flows, and subsequent flow bifurcations can occur before a chaotic state is reached. The dynamics are particularly rich in confined situations where geometry effects and boundary conditions play an important role and where symmetry considerations are involved [7]. Numerical linear stability analyses have first been carried out to determine the variation of $\mathrm{Ra}_{c}$ with the aspect ratio of the cavity, as those of Catton [8] for rectangular parallelepipedic cavities and Charlson and Sani [9] for cylindrical cavities. These linear stability studies were also able to identify the flow patterns that developed at values of the Rayleigh number, Ra, slightly above $\mathrm{Ra}_{c}$, but cannot give information about further flow transitions occurring at larger Ra. Nonlinear analyses were thus needed. The numerical methods using parameter continuation and bifurcation methods, which were recently developed in the case of three-dimensional situations, prove to be very efficient for such studies. In the cylindrical situation, we can mention the work on the influence of a free surface by Touihri et al. [10], where complex bifurcation diagrams have been obtained. In the parallelepipedic situation, very interesting results are reported by Puigjaner et al. [11-13]. Detailed bifurcation diagrams give the development of the steady flow patterns inside a cubical cavity heated from below with either adiabatic or conducting lateral walls and filled either with air $(\operatorname{Pr}=0.71)$ or silicone oil $(\operatorname{Pr}=130)$. Different flow patterns are found to be stable in the same Ra range. These bifurcation diagrams allow us to explain the transitions between different steady flow patterns observed experimentally by Pallares et al. [14] in a cubical cavity filled with silicone oil. Pallares et al. [15] also studied such transitions in a cubical cavity by three-dimensional direct numerical simulations. They obtain different flow structures and identify transitions between them, but cannot give precise bifurcation diagrams due to the lack of a continuation method.

Rayleigh-Bénard flows can be disturbed by small uncontrolled perturbations, coming for example from thermal defects or a small inclination of the cell (by an angle $\theta$ ). The inclination effect is particularly interesting as it induces a flow at any value of $\mathrm{Ra}$ and, thus, prevents the existence of the no-flow conductive state found in the horizontal case. Changes of flow symmetries will also occur and modifications of the bifurcation diagrams are expected. Rather few studies are related to this inclination effect in Rayleigh-Bénard situations. Cliffe and Winters [16] calculated the bifurcation diagram for a tilted two-dimensional square cavity. They show that the primary pitchfork bifurcation, which is found in the horizontal case, disappears and that the diagram predicts a leading solution (convective roll generated by the inclination) which develops 
smoothly from zero Rayleigh number and an anomalous solution (with opposite sense of rotation) on a disconnected branch initiated at a saddle-node point. The saddle-node critical Rayleigh number is also predicted as a function of the inclination angle of the cell. In their review paper on the Rayleigh-Bénard convection, Bodenschatz et al. [6] mention different studies concerned by the inclination effect. Most of these studies have considered extended layers and they analyze how the shear flow induced by the inclination modify the transitions to transverse or longitudinal rolls. One of the studies by Kirchartz and Oertel [17] considers more confined layers, with aspect ratios 10:4:1 and 4:2:1. Using both experiments and numerical calculations, the authors obtain very interesting results showing the influence of the inclination angle, the Rayleigh number, and the Prandtl number on the different flow patterns. More recently, Pallares et al. [15] give some information on the influence of very small inclinations $\left(\theta \leqslant 1^{\circ}\right)$ on the flow patterns obtained in a cubical cavity. At given values of $\mathrm{Ra}$, they show that some of the solutions obtained without inclination are still present with a small inclination, whereas other solutions are no longer observed and replaced by a solution with a different structure. They cannot, however, explain why these transitions occur. Finally, two other papers, a rather old paper by Ozoe et al. [18] and a quite recent paper by Adachi [19], consider the natural convection in an inclined square channel or duct. In these papers, the inclination is due to a rotation around the longitudinal axis of the channel. Ozoe et al. [18] performed both two-dimensional simulations in the cross section of the square channel and experiments in a convection channel with dimensions $15 \mathrm{~mm} \times 15 \mathrm{~mm} \times 270$ $\mathrm{mm}$. They focused on the heat transfer properties of natural convection expressed in terms of the Nusselt number. Both theoretically and experimentally, the maximum heat transfer is found to occur at about $50^{\circ}$ of inclination and is associated with a longitudinal roll. This longitudinal roll is obtained experimentally for inclinations $\theta>10^{\circ}$. For $\theta<10^{\circ}$, more complex flow structures are obtained, suggesting the presence of multiple stationary modes. Hence, a minimum heat transfer was found at an inclination of about $10^{\circ}$. Finally, when the square duct was kept almost horizontal $\left(\theta<1^{\circ}\right)$, a series of side-by-side two-dimensional roll cells was eventually established. Adachi [19] considers the stability of natural convection in an inclined square duct. He does not perform three-dimensional simulations, but considers that the duct has an infinite length and calculates the stability of the two-dimensional basic flows in the square cross section of the duct with regard to three-dimensional perturbations. He can thus obtain information on the longitudinal oneroll solution and on its stability, but cannot calculate the transverse roll solution, which is the first to appear without inclination, and cannot obtain the mixed mode solutions that are expected with inclination. We thus see that there is a need for three-dimensional simulations on such RayleighBénard problems involving tilted confined cavities, and that continuation methods would be the appropriate tool for these studies.

In this paper, a continuation method developed from a spectral finite element code is used to study the threedimensional flows in a tilted rectangular enclosure. We wanted to extend the former studies of Ozoe et al. [18] and Adachi
[19] on tilted square ducts to finite length cavities. Our rectangular enclosure with a length equal to two times the size of the square cross section can be considered as a truncated (or finite-length) square duct. This cavity has a reasonable length for three-dimensional computing, it avoids the solution degeneracy of the strongly symmetric cubical cavity, and, as in the duct with infinite length, the first transition is expected to be to transverse rolls and to occur before the transition to the longitudinal roll. The thermal boundary conditions on the sidewalls are chosen as adiabatic as in the work of Ozoe et al. [18]. On this problem, we first present the precise bifurcation diagram obtained in the horizontal situation. We then put into light all the subtle modifications induced in the bifurcation diagram around the first primary and secondary bifurcations by applying a slight tilt to the cavity. We finally determine the domains of existence for the different stable solutions in the $\mathrm{Ra}-\theta$ parameter space, and conclude with comparisons with previous works.

\section{MATHEMATICAL MODEL AND NUMERICAL TECHNIQUES}

\section{A. Mathematical model}

The mathematical model consists of a rectangular parallelepiped cavity filled with a fluid and differentially heated. The cavity has aspect ratios $A_{z}=L^{*} / h^{*}=2$ and $A_{y}=$ $l^{*} / h^{*}=1$, where $L^{*}$ is the length of the cavity (along $z^{*}$ ), $h^{*}$ is its height (along $x^{*}$ ), and $l^{*}$ is its width (along $y^{*}$ ). (Note that the superscript $*$ denotes the dimensional quantities.) The origin of the system of coordinates is placed at the center of the cavity and its axes are parallel to the edges of the cavity. The coordinates $\left(x^{*}, y^{*}, z^{*}\right)$ are then normalized by $h^{*}$ to obtain $(x, y, z)$, as shown schematically in Fig. 1 . The two walls corresponding to $y z$ planes at $x=-1 / 2$ and $x=1 / 2$ (lower and

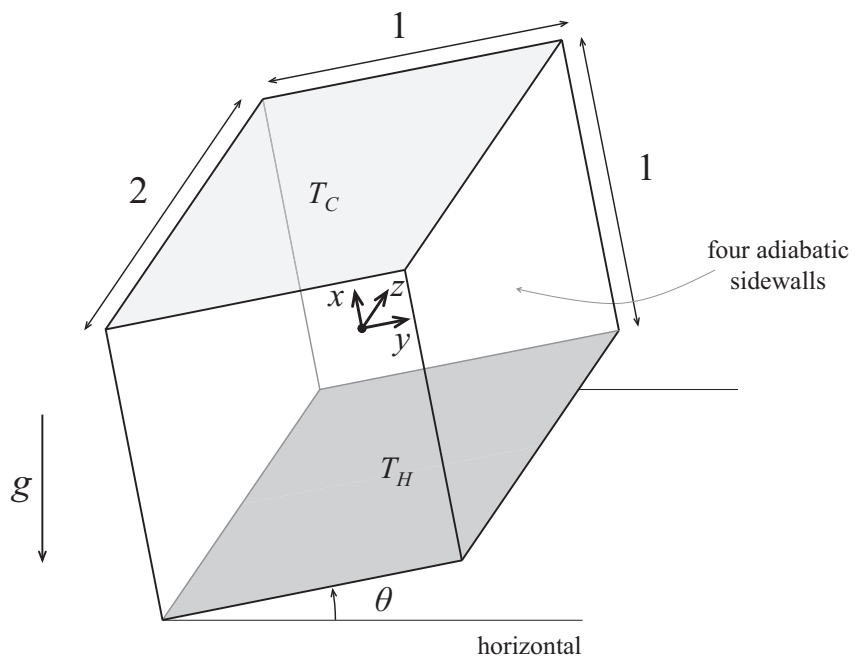

FIG. 1. Geometry of the dimensionless heated parallelepiped cavity. The $x y$ cross section of the cavity is a unit square and its length is 2 . The two walls corresponding to $y z$ planes at $x=-1 / 2$ and $x=1 / 2$ are isothermal and held at $T_{H}=1 / 2$ and $T_{C}=-1 / 2$, respectively. The other sidewalls are adiabatic. The cavity is tilted by an angle $\theta$ (expressed in degrees) around the $-z$ axis. 
upper walls, respectively, in the case without inclination) are isothermal. They are held at different temperatures, $T_{H}^{*}$ for the wall at $x=-1 / 2$ and $T_{C}^{*}$ for the wall at $x=1 / 2$, with $T_{H}^{*}>$ $T_{C}^{*}$, whereas the other sidewalls are adiabatic. The cavity is tilted by an angle $\theta$ around the $-z$ axis. $\theta$ is defined as the angle between the horizontal plane and the hot wall ( $y z$ plane at $x=$ $-1 / 2$ ) and is given in degrees throughout the text (the superscript $*$ is omitted). The fluid is assumed to be Newtonian with constant physical properties (kinematic viscosity $v^{*}$, thermal diffusivity $\kappa^{*}$, density $\rho^{*}$ ), except that, according to the Boussinesq approximation, the fluid density is considered as temperature dependent in the buoyancy term with a linear law $\rho^{*}=$ $\rho_{m}^{*}\left[1-\beta^{*}\left(T^{*}-T_{m}^{*}\right)\right]$, where $\beta^{*}$ is the thermal expansion coefficient and $T_{m}^{*}$ is a reference temperature taken as the mean temperature $\left(T_{H}^{*}+T_{C}^{*}\right) / 2$. The convective motions are then modeled by the Navier-Stokes equations coupled to an energy equation. Using $h^{*}, h^{* 2} / \kappa^{*}, \kappa^{*} / h^{*}, \rho^{*} \kappa^{* 2} / h^{* 2}$, and $\left(T_{H}^{*}-T_{C}^{*}\right)$ as scales for length, time, velocity, pressure, and temperature, respectively, these equations take the following form:

$$
\begin{gathered}
\nabla \cdot \boldsymbol{u}=0 \\
\frac{\partial \boldsymbol{u}}{\partial t}+(\boldsymbol{u} \cdot \nabla) \boldsymbol{u}=-\nabla p+\operatorname{Pr} \nabla^{2} \boldsymbol{u} \\
+\operatorname{Pr} \operatorname{Ra} T\left[\cos (\theta) \boldsymbol{e}_{x}+\sin (\theta) \boldsymbol{e}_{y}\right], \\
\frac{\partial T}{\partial t}+(\boldsymbol{u} \cdot \nabla) T=\nabla^{2} T,
\end{gathered}
$$

with boundary conditions given by $T=1 / 2$ on $x=-1 / 2$ and $T=-1 / 2$ on $x=1 / 2, \partial T / \partial z=0$ on $z=-1,1$ and $\partial T / \partial y=0$ on $y=-1 / 2,1 / 2$, and $\boldsymbol{u}=0$ on all the boundaries. The dimensionless variables are the velocity vector $\boldsymbol{u}=$ $(u, v, w)$, the pressure $p$, and the temperature $T=\left(T^{*}-T_{m}^{*}\right) /$ $\left(T_{H}^{*}-T_{C}^{*}\right) . \boldsymbol{e}_{x}$ and $\boldsymbol{e}_{y}$ are the unit vectors in the $x$ and $y$ directions, respectively, and the nondimensional parameters are the Rayleigh number, $\mathrm{Ra}=\beta^{*}\left(T_{H}^{*}-T_{C}^{*}\right) g h^{* 3} / \kappa^{*} \nu^{*}$, and the Prandtl number, $\operatorname{Pr}=\nu^{*} / \kappa^{*} . g$ is the gravitational acceleration. We can also define the Nusselt number $\mathrm{Nu}$, which expresses the actual heat transfer through $y z$ planes compared to the diffusive heat transfer. Due to the adiabatic sidewalls, $\mathrm{Nu}$ is the same for all $y z$ planes. It can be calculated easily at the boundaries $x= \pm 1 / 2$ where, due to the boundary condition $\boldsymbol{u}=0$, its expression is simply given by $\mathrm{Nu}=\int_{y, z}(-d T / d x) d y d z$.

\section{B. Numerical techniques}

The governing equations of the model are solved in the three-dimensional domain using a spectral element method, as described in [20]. The spatial discretization is obtained through Gauss-Lobatto-Legendre points distributions; the time discretization is carried out using a semi-implicit splitting scheme where, as proposed by Karniadakis et al. [21], the nonlinear terms are first integrated explicitly, the pressure is then solved through a pressure equation enforcing the incompressibility constraint (with a consistent pressure boundary condition derived from the equations of motion), and the linear terms are finally integrated implicitly. This time integration scheme is used for transient computations with the third-order accurate formulation described in [21]. But, in its first-order formulation, it is also used for steady state solving [22], eigenvalue and eigenvector calculation, and determination of bifurcation points $[23,24]$ through a Newton method. These methods, which are essential in this study to determine bifurcation diagrams, are described in the following.

We first consider steady state solving. The first order time scheme can be written in the abbreviated form

$$
\frac{\boldsymbol{X}^{(n+1)}-\boldsymbol{X}^{(n)}}{\Delta t}=\mathcal{N}\left(\boldsymbol{X}^{(n)}, \mathrm{Ra}\right)+\mathcal{L} \boldsymbol{X}^{(n+1)},
$$

where $\boldsymbol{X}$ denotes all of the spatially discretized fields $(\boldsymbol{u}(u, v, w), T)$, and $\mathcal{N}$ and $\mathcal{L}$ are the spatially discretized nonlinear and linear operators. For the purpose of the Newton solver, this time scheme can be slightly modified and expressed as

$$
\boldsymbol{X}^{(n+1)}-\boldsymbol{X}^{(n)}=-\mathcal{L}^{-1}\left[\mathcal{N}\left(\boldsymbol{X}^{(n)}, \mathrm{Ra}\right)+\mathcal{L} \boldsymbol{X}^{(n)}\right] .
$$

Now we consider the steady-state problem

$$
\mathcal{N}(\boldsymbol{X}, \mathrm{Ra})+\mathcal{L} \boldsymbol{X}=0,
$$

and solve it with a Newton method. Each Newton step can be written as

$$
\begin{aligned}
{\left[\mathcal{N}_{\boldsymbol{X}}(\boldsymbol{X}, \mathrm{Ra})+\mathcal{L}\right] \delta \boldsymbol{X} } & =-[\mathcal{N}(\boldsymbol{X}, \mathrm{Ra})+\mathcal{L} \boldsymbol{X}], \\
\boldsymbol{X} & \leftarrow \boldsymbol{X}+\delta \boldsymbol{X},
\end{aligned}
$$

where $\mathcal{N}_{\boldsymbol{X}}(\boldsymbol{X}, \mathrm{Ra})$ is the Jacobian of $\mathcal{N}$ with respect to $\boldsymbol{X}$ evaluated at $\boldsymbol{X}$ and $\mathrm{Ra}$. In order to improve the convergence of the iterative inversion, we rather solve

$$
-\mathcal{L}^{-1}\left[\mathcal{N}_{\boldsymbol{X}}(\boldsymbol{X}, \mathrm{Ra})+\mathcal{L}\right] \delta \boldsymbol{X}=-\left(-\mathcal{L}^{-1}\right)[\mathcal{N}(\boldsymbol{X}, \mathrm{Ra})+\mathcal{L} \boldsymbol{X}],
$$

where the operator $-\mathcal{L}^{-1}$ serves as a preconditioner (i.e., approximate inverse for $\mathcal{N}+\mathcal{L}$ ). If we solve the linear system (8) by an iterative conjugate gradient method, we need only provide the right-hand side and the action of the matrixvector product constituting the left-hand side. Referring to Eq. (5), we see that the right-hand side of Eq. (8) can be obtained by carrying out a time step, and the matrix-vector product by carrying out a linearized version of the same time step. The Jacobian matrix is thus never constructed or stored. The GMRES algorithm from the NSPCG software library [25] is generally used as iterative solver, as it was found to be more robust than the biconjugate gradient squared algorithm (BCGS) previously used [10]. Note that the system (8) is only appropriate for solving the steady state problem at constant $\mathrm{Ra}$. At a saddle node, where $\boldsymbol{X}$ ceases to be a function of Ra, a new algorithm is therefore needed to follow a solution branch. When any component $\boldsymbol{X}_{l}$ varies faster than some threshold, we treat this as an imminent saddle node by fixing $\boldsymbol{X}_{l}$ and allowing Ra to vary. One Newton step for solving (6) becomes

$$
\begin{aligned}
& {\left[\begin{array}{cc}
\mathcal{N}_{\boldsymbol{X}}(\boldsymbol{X}, \mathrm{Ra})+\mathcal{L} & \mathcal{N}_{\mathrm{Ra}}(\boldsymbol{X}, \mathrm{Ra}) \\
e_{l}^{T} & 0
\end{array}\right]\left[\begin{array}{c}
\delta \boldsymbol{X} \\
\delta \mathrm{Ra}
\end{array}\right]} \\
& \quad=-\left[\begin{array}{cc}
\mathcal{N}(\boldsymbol{X}, \mathrm{Ra})+\mathcal{L} \boldsymbol{X} \\
0
\end{array}\right], \\
& \boldsymbol{X} \leftarrow \boldsymbol{X}+\delta \boldsymbol{X}, \\
& \mathrm{Ra} \leftarrow \mathrm{Ra}+\delta \mathrm{Ra} .
\end{aligned}
$$


In this system, $e_{l}^{T}$ is the transpose of the $l$ th unit vector. This system will be preconditioned and solved in the same way as the system (7). With the use of (7) and (9), it will then be possible to follow any solution branches. The predictor, i.e., the initial guess for the Newton iteration, is evaluated by quadratic or linear extrapolation along the solution branch.

When computing steady solutions along a branch, we are interested in determining whether the solutions are stable or unstable. For that, we need to calculate the leading eigenvalues-those with the largest real part and thus responsible for initiating instability-and their corresponding eigenvectors. One possibility is to evaluate a set of those eigenvalues (for example the ten leading ones) with an Arnoldi method, which gives a large overview of the stability changes. To do so, we use Arnoldi's method from the ARPACK library [26] and follow the ideas of Mamun and Tuckerman [22], i.e., the action of the Jacobian is obtained by time stepping the linearized equations of the problem [linearized version of Eq. (5)] with a small time step equal to $10^{-5}$. In this way, we are able to calculate the exponential of the leading eigenvalues through Arnoldi's method with a good accuracy (the first ten real or complex leading eigenvalues and their corresponding eigenvectors are generally computed). Such Arnoldi calculation is, however, costly and cannot be done at each continuation step. Another possibility, using the fact that the eigenvalue spectrum usually does not change drastically when following a solution branch on a given range of the continuation parameter, is to follow a specific eigenvalue (generally the largest stable eigenvalue, which is expected to become soon unstable) at each continuation step through a Newton method. This calculation follows the same ideas as for steady-state solving. For a known steady solution $\boldsymbol{X}$, to calculate the eigenvalue $\lambda$ associated with a given eigenvector $\boldsymbol{h}$ whose $l$ th component is normalized to a given value $q$, we have to solve

$$
\begin{aligned}
{\left[\mathcal{N}_{X}(\boldsymbol{X}, \mathrm{Ra})+\mathcal{L}\right] \boldsymbol{h}-\lambda \boldsymbol{h} } & =0, \\
\boldsymbol{h}_{l}-q & =0 .
\end{aligned}
$$

One Newton step for solving the system (10) is

$$
\begin{aligned}
& {\left[\begin{array}{cc}
\mathcal{N}_{X}(\boldsymbol{X}, \mathrm{Ra})+\mathcal{L}-\lambda & -\boldsymbol{h} \\
\boldsymbol{e}_{l}^{T} & 0
\end{array}\right]\left[\begin{array}{l}
\delta \boldsymbol{h} \\
\delta \lambda
\end{array}\right]} \\
& \quad=-\left[\begin{array}{c}
{\left[\mathcal{N}_{X}(\boldsymbol{X}, \mathrm{Ra})+\mathcal{L}\right] \boldsymbol{h}-\lambda \boldsymbol{h}} \\
0
\end{array}\right], \\
& \boldsymbol{h} \\
& \qquad \boldsymbol{h}+\delta \boldsymbol{h}, \\
& \lambda \leftarrow \lambda+\delta \lambda .
\end{aligned}
$$

Preconditioned as in Eq. (8), this system can still be solved by conjugate gradient iterations, the different terms being obtained by minor modifications of the first-order time integration scheme. Note that $\lambda$ and $\boldsymbol{h}$ are real quantities in case of steady eigenvalue calculation, but they are complex quantities in case of oscillatory eigenvalue calculation. In this last case, we will rather write two real equations, respectively associated with the real part $\boldsymbol{h}_{r}$ and imaginary part $\boldsymbol{h}_{i}$ of the eigenvector.

Once we have identified a Ra range where a bifurcation point exists (the real part of an eigenvalue has been found to change its sign in this Ra range), we proceed to determine the precise location of this bifurcation point, i.e., find the corresponding critical Rayleigh number. This direct calculation of the bifurcation points is more complex than steady state solving but follows the same rationale. We will consider a steady bifurcation point. At this bifurcation point, $\boldsymbol{X}$ is a solution to Eq. (6), and the Jacobian is singular, with a null vector $\boldsymbol{h}$ whose $l$ th component will be normalized to $q$ :

$$
\begin{aligned}
\mathcal{N}(\boldsymbol{X}, \mathrm{Ra})+\mathcal{L} \boldsymbol{X} & =0, \\
{\left[\mathcal{N}_{\boldsymbol{X}}(\boldsymbol{X}, \mathrm{Ra})+\mathcal{L}\right] \boldsymbol{h} } & =0, \\
\boldsymbol{h}_{l}-q & =0 .
\end{aligned}
$$

One Newton step for solving Eqs. (12) is

$$
\begin{aligned}
& {\left[\begin{array}{ccc}
\mathcal{N}_{\boldsymbol{X}}(\boldsymbol{X}, \mathrm{Ra})+\mathcal{L} & 0 & \mathcal{N}_{\mathrm{Ra}}(\boldsymbol{X}, \mathrm{Ra}) \\
\mathcal{N}_{\boldsymbol{X}, \boldsymbol{X}}(\boldsymbol{X}, \mathrm{Ra}) \boldsymbol{h} & \mathcal{N}_{\boldsymbol{X}}(\boldsymbol{X}, \mathrm{Ra})+\mathcal{L} & \mathcal{N}_{\boldsymbol{X}, \mathrm{Ra}}(\boldsymbol{X}, \mathrm{Ra}) \boldsymbol{h} \\
0 & e_{l}^{T} & 0
\end{array}\right]} \\
& {\left[\begin{array}{c}
\delta \boldsymbol{X} \\
\delta \boldsymbol{h} \\
\delta \mathrm{Ra}
\end{array}\right]=-\left[\begin{array}{c}
\mathcal{N}(\boldsymbol{X}, \mathrm{Ra})+\mathcal{L} \boldsymbol{X} \\
{\left[\mathcal{N}_{\boldsymbol{X}}(\boldsymbol{X}, \mathrm{Ra})+\mathcal{L}\right] \boldsymbol{h}} \\
0
\end{array}\right],} \\
& \boldsymbol{X} \leftarrow \boldsymbol{X}+\delta \boldsymbol{X}, \\
& \boldsymbol{h} \leftarrow \boldsymbol{h}+\delta \boldsymbol{h}, \\
& \mathrm{Ra} \leftarrow \mathrm{Ra}+\delta \text { Ra. }
\end{aligned}
$$

Note that for a primary bifurcation point, $\boldsymbol{X}$ is the known conductive solution, and the Newton step is reduced to

$$
\begin{aligned}
& {\left[\begin{array}{cc}
\mathcal{N}_{\boldsymbol{X}}(\boldsymbol{X}, \mathrm{Ra})+\mathcal{L} & \mathcal{N}_{X, \mathrm{Ra}}(\boldsymbol{X}, \mathrm{Ra}) \boldsymbol{h} \\
e_{l}^{T} & 0
\end{array}\right]\left[\begin{array}{c}
\delta \boldsymbol{h} \\
\delta \mathrm{Ra}
\end{array}\right]} \\
& \quad=-\left[\begin{array}{c}
{\left[\mathcal{N}_{\boldsymbol{X}}(\boldsymbol{X}, \mathrm{Ra})+\mathcal{L}\right] \boldsymbol{h}} \\
0
\end{array}\right], \\
& \boldsymbol{h} \leftarrow \boldsymbol{h}+\delta \boldsymbol{h}, \\
& \mathrm{Ra} \leftarrow \mathrm{Ra}+\delta \mathrm{Ra} .
\end{aligned}
$$

In the systems (13) and (14), $\mathcal{N}_{\mathbf{R a}}$ is the Jacobian of $\mathcal{N}$ with respect to $\mathrm{Ra}, \mathcal{N}_{\boldsymbol{X}, \boldsymbol{X}}$ is the double Jacobian of $\mathcal{N}$ with respect to $\boldsymbol{X}$, and $\mathcal{N}_{\boldsymbol{X}, \mathbf{R a}}$ is the Jacobian of $\mathcal{N}$ with respect to both $\boldsymbol{X}$ and Ra. Preconditioned as in Eq. (8), these systems can still be solved by conjugate gradient iterations. As before, the different terms of the right-hand side and of the matrixvector product are obtained through slightly adapted first order normal or linearized time steps. Note that the direct calculation of Hopf bifurcation points, which follows the same ideas, is also available in the code, but it was almost not used during this study.

\section{Continuation}

Our first objective is to compute the global bifurcation diagrams associated with our physical system. For that, a continuation procedure has been developed, which organizes the different steps of the calculation. Note that we have first to define the range of Ra values over which we want to follow the solution branches.

We can take the noninclined situation, where a diffusive solution exists, as an example. In that case, the leading eigenvalues of the diffusive solution are first calculated for increasing Ra in order to detect the steady primary bifurcations. We alternate an expensive step using Arnoldi's method 
allowing the calculation of the ten leading eigenvalues and several cheaper steps based on the solution of the system (11) allowing us to follow a specific, well chosen eigenvalue. When the real part of an eigenvalue changes sign as $\mathrm{Ra}$ is increased, this is an indication that a bifurcation just occurred. The corresponding eigenvector is thus used as an initial guess in the direct calculation of the bifurcation point, which is performed by solving the Newton system (14). The critical Rayleigh number, $\mathrm{Ra}_{c}$, and the critical eigenvector are then stored. Once the limit Ra number is reached, a new sequence begins.

For each primary bifurcation point, successively, a branching algorithm uses the critical eigenvector as a predictor in order to jump to the steady solution branch emerging at this point. This branching algorithm is based on the solution of the system (9) so as to be able to catch both supercritical and subcritical branches. The new branch is then followed by continuation, using either (7) or (9), up to the limit Ra number. For each steady solution calculated along the branch, some leading eigenvalues are calculated, in the same way as for the diffusive solution branch. This allows us to detect the secondary steady bifurcation points, which, once detected, will be precisely calculated using (13), with an initial guess corresponding to the solution and the transitional eigenvector at the detection point. The steady branches emerging at the steady secondary bifurcation points can, in turn, be reached using the branching algorithm, with a predictor built on the critical solution perturbed by the critical eigenvector. These branches can then be followed by continuation up to the limit Ra number.

The direct calculation of the bifurcation points by Newton method can also be used independently. When a bifurcation point has been found to play an important role in the dynamics of our system, it could be interesting to follow its evolution as we vary other parameters of the problem (for example, the inclination angle or the Prandtl number). In that case, the critical Rayleigh number, solution, and eigenvector at the reference value of the parameter are used as initial guess, and the parameter is varied with a small increment. When the convergence of the Newton system [(14) or (13)] at a given value of the parameter is reached, new critical values are obtained, which, in turn, are used as the initial guess for the next step at a slightly modified value of the parameter. We generally choose a constant increment value for the parameter, but this value is further decreased if difficulties of convergence are encountered. Stability maps can thus be obtained, which may improve the understanding of our physical situation by extending the results obtained with the bifurcation diagrams at given parameter values to larger parameter ranges.

Note that, in spite of the continuation procedure, it is difficult to calculate a complex bifurcation diagram in a single run of the code. The fact that, at each continuation step, we do not use Arnoldi's method, but rather follow a selected eigenvalue, makes it possible to miss some of the bifurcations. We can indeed follow an eigenvalue, which seemed to be a good candidate for transition, while another eigenvalue evolves more quickly and is eventually the first to become unstable. Sometimes, this transition can be detected at the next Arnoldi step. In any case, an a posteriori careful reading of the output file will reveal any missed bifurcations, and a specific calculation will then be conducted to determine them precisely. We have, however, tested that the bifurcation diagram corresponding to the horizontal cavity in the range $0 \leqslant \mathrm{Ra} \leqslant 4000$ (see Fig. 2) could be obtained in a single run of the code.

\section{RESULTS}

Our results concern the convective flows induced in a parallelepiped cavity with $A_{y}=1$ (square $x y$ cross section) and $A_{z}=2$, and for $\operatorname{Pr}=1$. After some validation tests and symmetry considerations, we will first consider the case where the cavity is horizontal, and we will then study the influence of an inclination of the cell, when this inclination corresponds to a rotation around the longest axis of the cell (here the $-z$ axis).

\section{A. Validation and tests of accuracy}

The validation of the method cannot be done with the parallelepiped cavity studied here as no results are available for such cavity. We then choose the case of the cubical cavity which was recently studied by Puigjaner et al. [11-13]. Table I shows the critical Rayleigh number, $\mathrm{Ra}_{c}$, obtained for the first three primary bifurcations inside a cubical cavity $\left(A_{y}=1\right.$, $A_{z}=1$ ) heated from below. The calculations were conducted for $\operatorname{Pr}=1$ and for three different numbers of grid points. We first see the good convergence of the results with the refinement of the grid. The critical Rayleigh number obtained for the same primary bifurcation points by Puigjaner et al. [12] inside a cubical cavity for $\operatorname{Pr}=130$ are also given for comparison. (Note that $\mathrm{Ra}_{c}$ corresponding to a primary threshold does not depend on Pr.) A good agreement is obtained between the different results. In order to also assess the accuracy of the code at higher Ra, as well as the feasibility of the calculation when the cavity is heated from the side, we ran the code for the well-known case of a cubical cavity filled with air $(\operatorname{Pr}=0.71)$ differentially heated between two opposite vertical sidewalls $\left(\theta=90^{\circ}\right)$ with the remaining walls set adiabatic. A benchmark for this situation was proposed by Wakashima et al. [27]. They computed the Nusselt number $\mathrm{Nu}$ in this cubical cavity with a finite difference method having a fourth-order accuracy. Table II compares the Nusselt number obtained by our continuation method to the benchmark values. We used a $31 \times 31 \times 31$ grid with a Gauss-Lobatto-Legendre point distribution while Wakashima et al. [27] used a uniform $120 \times 120 \times 120$ grid. As expected, the differences between the benchmark values and our results increase at higher values of Ra. Nevertheless, the percent difference is within $0.7 \%$ even at values of $\mathrm{Ra}$ as high as $10^{6}$.

For the calculations in the parallelepiped cavity $\left(A_{y}=1\right.$, $A_{z}=2$ ), the same refined mesh comprising $27 \times 27 \times 41$ points (in the $x, y$, and $z$ directions, respectively) was chosen for all the cases. It gives a very good precision for the calculation of the different bifurcation points, as indicated by the precision tests given in Table III. (The bifurcation points used in these mesh refinement tests are presented further in this section.) The precision slightly decreases for inclined cavities when the variation of the critical Rayleigh number with $\theta$ is very steep (see Fig. 8). The test done on the saddle-node point $N \pm$ for $\theta=4^{\circ}$, however, shows that the value of $\operatorname{Ra}_{c}$ changes by less than $0.01 \%$ when the mesh is further refined. 


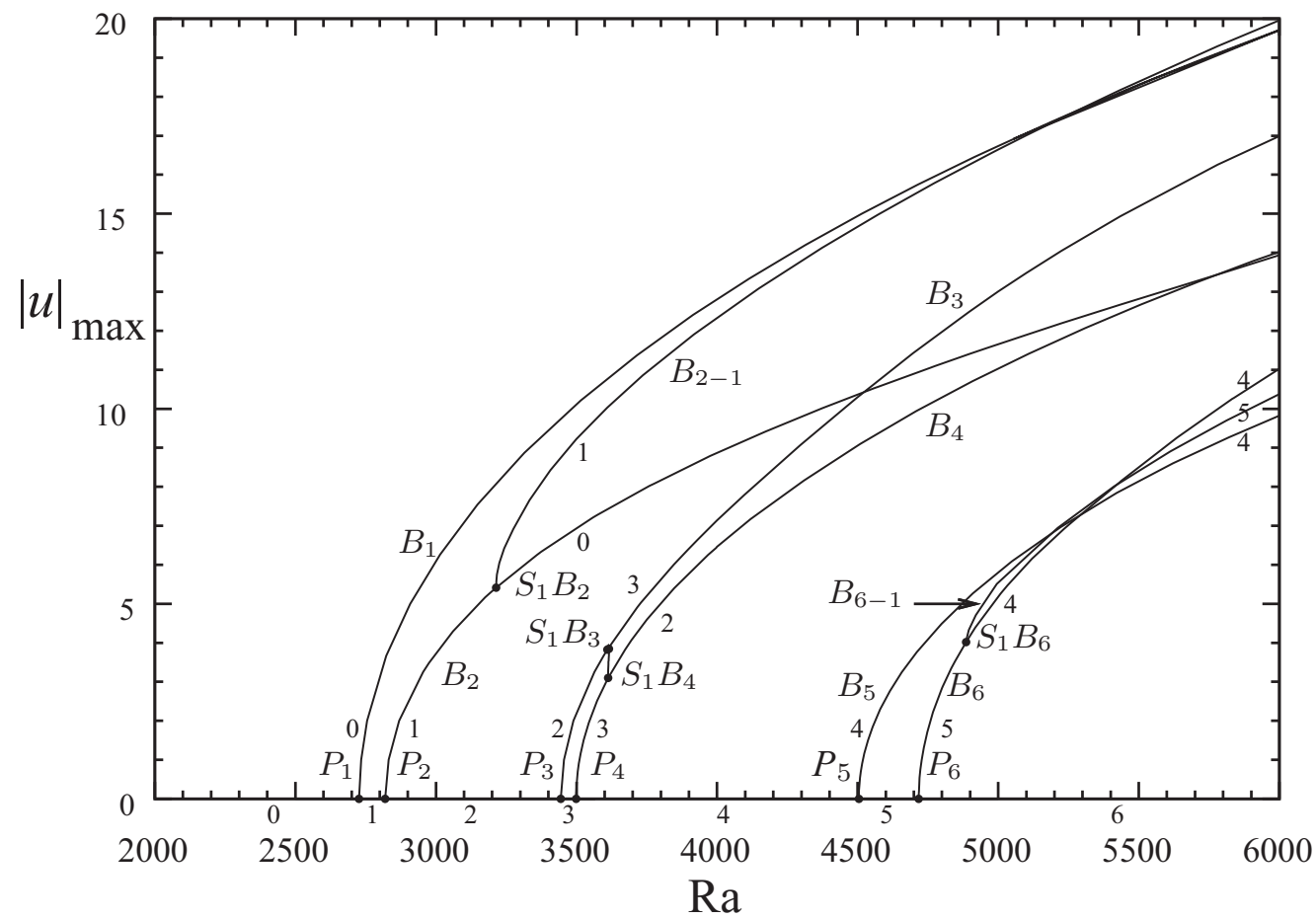

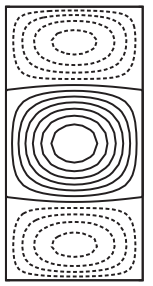

$P_{1}$

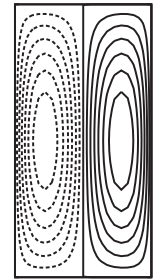

$P_{2}$

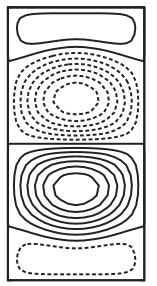

$P_{3}$

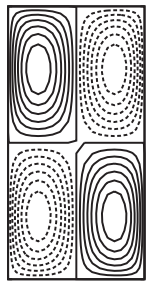

$P_{4}$

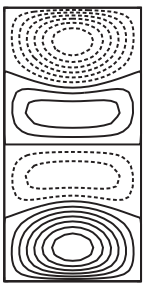

$P_{5}$

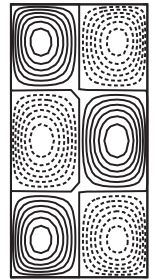

$P_{6}$

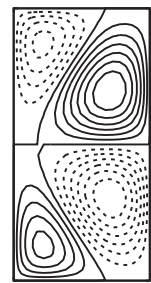

$S_{1} B_{3-1}$

FIG. 2. Bifurcation diagram in the case of a horizontal $1 \times 1 \times 2$ cavity heated from below $(\operatorname{Pr}=1)$. The abscissa indicates the Rayleigh number $\mathrm{Ra}$ and the ordinate the maximum absolute value of the vertical velocity inside the cavity. The solution branches $B_{j}$ obtained in the range $2000 \leqslant \mathrm{Ra} \leqslant 6000$ and initiated from the first six primary bifurcations are plotted. The number of eigenvalues with a positive real part (unstable steady eigenvalues for this diagram) is indicated for each branch, 0 corresponding to the stable solutions. Solid circles indicate steady bifurcation points denoted as $P_{i}$ (for the $i$ th primary point) or $S_{i} B_{j}$ (for the $i$ th secondary bifurcation point on the branch $B_{j}$ ). Insets show vertical velocity contours in the horizontal midplane $(x=0)$ for the critical eigenvectors at the first six primary bifurcation points $\left(P_{1}\right.$ to $\left.P_{6}\right)$ and for the solution at the saddle-node point $S_{1} B_{3-1}$ on the closed loop branch $B_{3-1}$ between $S_{1} B_{3}$ and $S_{1} B_{4}$. The positive and negative vertical velocities are indicated by solid and dashed lines, respectively.

The calculation of the solutions by the Newton method is less difficult, so that the chosen mesh also gives a good precision for the solutions in the whole parameter range studied $\left(0 \leqslant \mathrm{Ra} \leqslant 25000,0^{\circ} \leqslant \theta \leqslant 90^{\circ}\right)$.

\section{B. Symmetries}

In such confined convective situations, the flow states have different symmetries which play an important role as they are often involved in the different bifurcations. Indeed, at

TABLE I. Critical Rayleigh numbers $\mathrm{Ra}_{c}$ for the first three primary bifurcation points $\left(P_{1}, P_{2}\right.$, and $\left.P_{3}\right)$ in a horizontal cubical cavity heated from below $\left(\theta=0^{\circ}\right)$. Three different meshes are listed to assess the calculation accuracy. The Prandtl number is set to $\operatorname{Pr}=1$. The results of Puigjaner et al. [12] are listed for comparison $(\operatorname{Pr}=130)$.

\begin{tabular}{lcccr}
\hline \hline & This work, $\operatorname{Pr}=1$ & & Puigjaner et al. [12] \\
\hline Grid & $22 \times 22 \times 22$ & $27 \times 27 \times 27$ & $33 \times 33 \times 33$ & Pr $=130$ \\
\hline$P_{1}$ & 3388.521 & 3388.525 & 3388.527 & 3389 \\
$P_{2}$ & 5900.429 & 5900.442 & 5900.448 & 5902 \\
$P_{3}$ & 7456.133 & 7456.143 & 7456.148 & 7458 \\
\hline \hline
\end{tabular}


TABLE II. Nusselt number $\mathrm{Nu}$ in a cubical cavity filled with air $(\operatorname{Pr}=0.71)$ differentially heated between two opposite vertical sidewalls $\left(\theta=90^{\circ}\right)$ at high values of Ra. Comparisons between our results and the benchmark results proposed by Wakashima et al. [27].

\begin{tabular}{cccc}
\hline \hline $\mathrm{Ra}$ & $\begin{array}{c}\text { This study, } \\
\mathrm{Nu}\end{array}$ & $\begin{array}{c}\text { Wakashima et al. [27], } \\
\mathrm{Nu}\end{array}$ & $\begin{array}{c}\text { Difference, } \\
\Delta \mathrm{Nu}(\%)\end{array}$ \\
\hline $10^{4}$ & 2.0551 & 2.0624 & $0.0073(0.35)$ \\
$10^{5}$ & 4.3370 & 4.3665 & $0.0295(0.68)$ \\
$10^{6}$ & 8.6394 & 8.6973 & $0.0579(0.67)$ \\
\hline \hline
\end{tabular}

a bifurcation point, a symmetry of the solution is generally broken by the critical eigenvector (pitchfork bifurcation) and the solutions on the new branch bifurcating at this point will have lost this symmetry. Checking the symmetries is then not only important to characterize the different flow states, but also to check the coherence of the bifurcation diagram and sometimes to guess the new solutions which must be obtained (for example, when a tilt of the cavity is imposed).

Under the approximation of the model, the basic diffusive no-flow solution in the horizontal situation (which is simply $T=-x$ and $\boldsymbol{u}=0$ ) presents different symmetries: reflection symmetries $S_{P_{y z}}, S_{P_{x z}}$, and $S_{P_{x y}}$ with respect to the three middle planes (longitudinal $y z$ plane at $x=0$, longitudinal $x z$ plane at $y=0$, and transverse $x y$ plane at $z=0$, respectively), which, by combination, induce $\pi$-rotational symmetries $S_{A_{x}}$, $S_{A_{y}}$, and $S_{A_{z}}$ about the three middle axes ( $x, y$, and $z$ axes, respectively). These symmetries belong to a $Z_{2} \times Z_{2} \times Z_{2}=$ $Z_{2} \times D_{2}$ group. As an example, we define two of these symmetries, $S_{P_{x y}}$ and $S_{A_{z}}$, and the others can be obtained by circular permutation:

$$
\begin{aligned}
S_{P_{x y}} & :(x, y, z, t) \rightarrow(x, y,-z, t), \\
(u, v, w, T) & \rightarrow(u, v,-w, T), \\
S_{A_{z}} & :(x, y, z, t) \rightarrow(-x,-y, z, t), \\
(u, v, w, T) & \rightarrow(-u,-v, w,-T) .
\end{aligned}
$$

The symmetry $S_{C}$ with respect to the center point of the cavity can also be obtained by combination of the previous symmetries. When the flow is triggered, at least the up-down $S_{P_{y z}}$ symmetry is broken. The effective symmetries of the flow will depend on the flow configuration triggered.

When the cavity is tilted around the longitudinal $-z$ axis, there is no motionless state for $\mathrm{Ra} \neq 0$ and the convection first induced corresponds to a single longitudinal roll with the $-z$ axis as axis of rotation and the same sense of rotation as the inclination angle. The original symmetries of this inclined
TABLE IV. Critical Rayleigh number $\mathrm{Ra}_{c}$ for the first six primary bifurcation points $\left(P_{1}-P_{6}\right)$ for a horizontal $1 \times 1 \times 2$ cavity heated from below $(\operatorname{Pr}=1)$.

\begin{tabular}{lccccc}
\hline \hline$P_{1}$ & $P_{2}$ & $P_{3}$ & $P_{4}$ & $P_{5}$ & $P_{6}$ \\
\hline 2726.53 & 2818.78 & 3443.54 & 3498.72 & 4504.21 & 4717.45 \\
\hline \hline
\end{tabular}

case are those of the longitudinal roll, which are the $S_{P_{x y}}$, $S_{A_{z}}$, and $S_{C}$ symmetries. These symmetries belong to a $Z_{2} \times$ $Z_{2}=D_{2}$ group. In both the horizontal and tilted situations, when increasing $\mathrm{Ra}$, bifurcations to new flow states (steady or oscillatory) will occur, at which some of the symmetries will usually be broken.

\section{Horizontal cavity}

In the case of the horizontal cavity, where a diffusive solution exists and where convection sets in beyond primary bifurcation points, the entire continuation algorithm described in Sec. II C was used to calculate the bifurcation diagram. All the solutions emerging from the first ten primary bifurcation points and within the range $0 \leqslant \mathrm{Ra} \leqslant 10000$ were calculated. For reasons of concision, we will restrict the detailed presentation to the solutions in the range $0 \leqslant \mathrm{Ra} \leqslant 6000$, which emerge from the first six primary bifurcation points (denoted as $P_{1}$ to $P_{6}$ ). The onset of convection, at the first primary bifurcation point $P_{1}$, occurs at $\mathrm{Ra}=2726.53$. The critical values for the other five primary bifurcation points can be found in Table IV. All these primary bifurcations are pitchforks, as they at least break the up-down symmetry.

The critical eigenvectors at these primary bifurcation points are plotted as insets in Fig. 2. Here, the vertical velocity contours in the horizontal midplane $(x=0)$ are presented, solid and dashed lines indicating positive and negative vertical velocities. We see that the eigenstructures can be characterized by their number $n_{T}$ of counter-rotating transverse rolls (with axis along $y$ ) and their number $n_{L}$ of counter-rotating longitudinal rolls (with axis along $z$ ), and can then be denoted as $\left(n_{T}, n_{L}\right)$ structures. They also have characteristic symmetries which can be related to the parity of $n_{T}$ or $n_{L}$ : odd values correspond to symmetries with respect to axes and even values correspond to symmetries with respect to planes. We thus see for example that the critical eigenstructure at $P_{1}[(2,0)$ structure] has the $S_{P_{x y}}$ and $S_{P_{x z}}$ symmetries, whereas those at $P_{2}[(0,1)$ structure $]$ and $P_{6}[(2,1)$ structure $]$ have the $S_{P_{x y}}$ and $S_{A_{z}}$ symmetries.

The bifurcation diagram in the range $\mathrm{Ra} \leqslant 6000$ is presented in Fig. 2. The maximum absolute value of the vertical velocity $|u|_{\max }$ on the grid is plotted as a function of the

\begin{tabular}{|c|c|c|c|c|c|c|}
\hline Grid points along $x, y$, and $z$ & $P_{1}$ & $P_{6}$ & $S_{1} B_{2}$ & $S_{1} B_{6}$ & $S_{d}\left(\theta=7^{\circ}\right)$ & $N \pm\left(\theta=4^{\circ}\right)$ \\
\hline $23 \times 23 \times 37$ & 2726.521 & 4717.442 & 3213.635 & 4885.286 & 10584.305 & 16916.910 \\
\hline $27 \times 27 \times 41$ & 2726.525 & 4717.450 & 3213.618 & 4885.294 & 10584.236 & 16919.437 \\
\hline $31 \times 31 \times 45$ & 2726.528 & 4717.453 & 3213.610 & 4885.298 & 10584.210 & 16920.352 \\
\hline
\end{tabular}

TABLE III. Mesh refinement tests of numerical accuracy for different critical Rayleigh numbers corresponding to primary bifurcation points $\left(P_{1}\right.$ and $\left.P_{6}\right)$ and secondary bifurcation points $\left(S_{1} B_{2}\right.$ and $\left.S_{1} B_{6}\right)$ for $\theta=0^{\circ}$, to the secondary bifurcation point $S_{d}$ for $\theta=7^{\circ}$, and the saddle-node point $N \pm$ for $\theta=4^{\circ}$. 


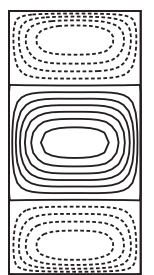

$B_{1}$

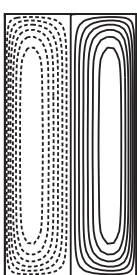

$B_{2}$

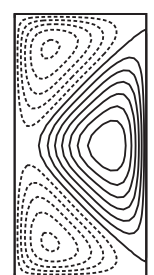

$B_{2-1}$

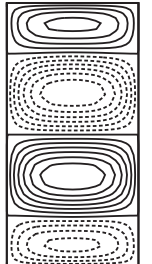

$B_{3}$

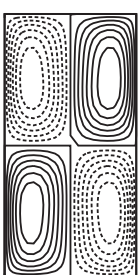

$B_{4}$

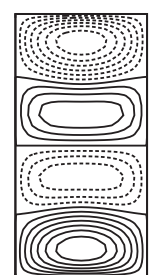

$B_{5}$

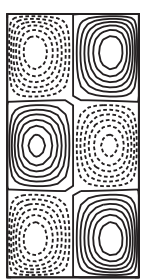

$B_{6}$

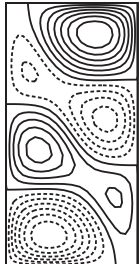

$B_{6-1}$

FIG. 3. Vertical velocity contours in the horizontal midplane $(x=0)$ for the solutions at $\mathrm{Ra}=6000$ on the different branches issued from the first six primary bifurcation points for a horizontal $1 \times 1 \times 2$ cavity heated from below $(\operatorname{Pr}=1)$. Among these eight solutions, only the solutions on the $B_{1}$ and $B_{2}$ branches are stable.

Rayleigh number Ra. For this diagram, we only calculated one branch emerging from each bifurcation point. This, however, is not a restriction in the horizontal case as we will see that all the bifurcations (as the primary bifurcations) are pitchfork bifurcations, for which the two emerging branches give equivalent solutions and can be plotted as a single curve in our diagram featuring $|u|_{\max }$. Such a diagram could have been equivalently plotted using the Nusselt number $\mathrm{Nu}$. The plot with $|u|_{\max }$ was clearer and was then preferred. In the diagram, precisions on the stability of each branch are given by indicating the number of unstable real eigenvalues (no unstable complex conjugate eigenvalues were found). Solid circles indicate steady bifurcation points. Note moreover that all the solutions obtained on the different branches at $\mathrm{Ra}=6000$ are depicted in Fig. 3. In this range $(\mathrm{Ra} \leqslant 6000)$, six primary bifurcations were detected on the diffusive branch and precisely calculated. From these primary bifurcations, thanks to the branching algorithm, six primary branches were calculated and followed up to $\mathrm{Ra}=6000$. Four secondary bifurcation points were then detected and precisely calculated on these primary branches, and the secondary branches emerging from these points were eventually calculated. We choose to denote the primary and secondary branches as $B_{i}$ and $B_{i-j}$, respectively, where $i$ is the index of a primary branch and $j$ is the index of a secondary branch on this primary branch. Likewise, the secondary bifurcation points on these branches (say $B_{m}$ ) will be denoted as $S_{l} B_{m}$, where $l$ indicates the $l$ th secondary bifurcation point. The results show that the first primary branch $B_{1}$, which corresponds to two transverse rolls, is stable in the calculated range of $\mathrm{Ra}$. The second primary branch $B_{2}$, which corresponds to a single longitudinal roll, is unstable at its onset, but stabilized at a secondary bifurcation point $S_{1} B_{2}\left(\mathrm{Ra}_{c}=3213.62\right)$. The critical eigenvector at this bifurcation point is a transverse two-roll structure, similar to the primary eigenvector at $P_{1}$. The bifurcated branch $B_{2-1}$, which is one time unstable, corresponds to a kind of two-oblique-roll structure which has kept the reflection symmetry with respect to the $x y$ plane, as shown in Fig. 3. The third primary branch $B_{3}$ corresponding to three transverse rolls and the fourth primary branch $B_{4}$ corresponding to a $(1,1)$ structure (which are, respectively, two-time and three-time unstable at their onset) exchange stability through a short secondary branch which connects them. This branch (which can be denoted as $B_{3-1}$ or $B_{4-1}$ ) bifurcates supercritically from $B_{3}$ at $S_{1} B_{3}\left(\mathrm{Ra}_{c}=3609.24\right)$, turns at a saddle-node point $S_{1} B_{3-1}\left(\mathrm{Ra}_{c}=3615.53\right)$, and connects with $B_{4}$ at $S_{1} B_{4}$
$\left(\mathrm{Ra}_{c}=3612.51\right)$. The eigenvector at the secondary bifurcation point on $B_{3}\left(B_{4}\right)$ is similar to the flow solution on $B_{4}\left(B_{3}\right)$. The structure of the flow on this closed loop secondary branch is shown as the inset in Fig. 2 at the saddle-node point $S_{1} B_{3-1}$. We see that this structure still includes oblique rolls, but with a $\pi$-rotation symmetry with respect to the $y$ axis. Note that this secondary branch was calculated two times during the continuation procedure, as it emerges from the secondary bifurcation points on both $B_{3}$ and $B_{4}$. The fifth primary branch $B_{5}$, which corresponds to a long-scale transverse circulation embedding two co-rotating transverse cells, remains four-time unstable from its onset at $P_{5}$ to $\mathrm{Ra}=6000$. The sixth primary branch $B_{6}$ (five-time unstable at its onset and corresponding to a $(2,1)$ structure $)$ is stabilized once at $S_{1} B_{6}\left(\mathrm{Ra}_{c}=4885.29\right)$. The solution on the bifurcated branch $B_{6-1}$, which is five time unstable, has lost the $S_{P_{x y}}$ symmetry.

A summary of the characteristics of this bifurcation diagram is finally given in Table $\mathrm{V}$ and Fig. 3. In Table V, we give the values of the critical Rayleigh number for the secondary bifurcations involved in the diagram. Five such bifurcations have been found, among which four are secondary pitchforks and one is a saddle node. These bifurcation points were directly calculated as the solution of the system (13), which allows us to obtain results with a very good precision. In Fig. 3, the eight solutions obtained at $\mathrm{Ra}=6000$ on the different branches issued from the first six primary bifurcation points are depicted. Note however that, as these branches are initiated at pitchfork bifurcation points, an equivalent solution exists for each of these solutions, which is its symmetric with respect to the symmetry broken at the pitchfork point. The solutions on the six primary branches $\left(B_{1}\right.$ to $\left.B_{6}\right)$ are similar to the eigenvectors that initiate them (at points $P_{1}$ to $P_{6}$, respectively) and have the same symmetries. The convective flows are, however, more intense, so that thinner boundary layers are observed. For the solutions on the secondary branches, one of the symmetries

TABLE V. Critical Rayleigh number $\mathrm{Ra}_{c}$ for the secondary bifurcation points found in the range $\mathrm{Ra} \leqslant 6000$ for a horizontal $1 \times 1 \times 2$ cavity heated from below $(\operatorname{Pr}=1) . S_{i} B_{j}$ denotes the $i$ th secondary bifurcation point on the branch $B_{j}$. All these secondary bifurcation points are pitchforks, except $S_{1} B_{3-1}$ which is a saddlenode point connecting $B_{3-1}$ to $B_{4-1}$.

\begin{tabular}{lcccc}
\hline \hline$S_{1} B_{2}$ & $S_{1} B_{3}$ & $S_{1} B_{3-1}$ & $S_{1} B_{4}$ & $S_{1} B_{6}$ \\
\hline 3213.62 & 3609.24 & 3615.53 & 3612.51 & 4885.29 \\
\hline \hline
\end{tabular}


of the corresponding primary solutions has been broken. For example, the $S_{A_{z}}$ symmetry of $B_{2}$ is broken on $B_{2-1}$ which keeps the $S_{P_{x y}}$ symmetry, and the $S_{P_{x y}}$ and $S_{A_{z}}$ symmetries of $B_{6}$ are broken on $B_{6-1}$ which keeps the central $S_{C}$ symmetry. Further calculations extended up to $\mathrm{Ra}=10000$ and initiated from the first ten primary bifurcations have shown that up to 18 secondary and tertiary bifurcations can be found and that 22 solutions are obtained at $\mathrm{Ra}=10000$. Nevertheless, among all these solutions, only the first two solutions on $B_{1}$ and $B_{2}$ are stable. The solution on the $B_{1}$ branch corresponds to a transverse two-roll structure (denoted as a $T$ solution) and is stable at its onset at $P_{1}\left(\mathrm{Ra}_{c}=2726.53\right)$. The solution on the $B_{2}$ branch corresponds to a longitudinal one-roll structure (denoted as a $L$ solution) and is stable beyond the secondary bifurcation $S_{1} B_{2}\left(\mathrm{Ra}_{c}=3213.62\right)$. Additional computations for larger $\mathrm{Ra}$ values were performed for these two branches. The branches appear to be stable in a large Ra range, at least up to $\mathrm{Ra}=80000$. Their stability limits correspond to Hopf bifurcation points, which have been localized at $\mathrm{Ra}_{c}=87025$ and 81156 for the $B_{1}$ and $B_{2}$ branches, respectively. The associated angular frequencies are $\omega=154.43$ and 8.72.

Note finally that the degeneracy of the solutions at the first bifurcation point in a cubical cavity (due to the $D_{4}$ symmetry, see Puigjaner et al. [13]) is suppressed when $A_{z} \neq A_{y}$ (change to a $D_{2}$ symmetry). In our cavity with $A_{z}=2$ and $A_{y}=1$, the $T, L$ and oblique solutions, which were triggered at the same bifurcation point in the cubical cavity, are now separated. More precisely, the $T$ and $L$ solutions still belong to primary branches (as they have the $D_{2}$ symmetries), the $T$ solution being the first to appear, whereas oblique solutions can be found on a secondary branch initiated from the $L$ branch. This sequence of primary bifurcations ( $T$ solutions triggered before $L$ solutions) is similar to that found in longer or infinite square ducts [18,19]. This is an argument for calling our parallelepiped cavity a truncated or finite length square duct and we can expect that the comparisons between our results and those in longer square ducts will make sense.

\section{Tilted cavity}

In this part of the study concerned by the influence of a tilt $\theta$ of the cavity around the horizontal $-z$ axis, we will focus on the two branches which are stable (at least in part) in the horizontal situation (the $B_{1}$ and $B_{2}$ branches). As indicated in Sec. III B, when a tilt is imposed to the cavity, the symmetries of the problem are changed and the bifurcations found in the horizontal situation will change as well. To better analyze the influence of the tilt, we will compare the bifurcation diagram obtained in the horizontal situation $\left(\theta=0^{\circ}\right)$ with that obtained for a very small inclination angle, $\theta=0.01^{\circ}$. The two calculated diagrams are plotted in Fig. 4 in the same $\mathrm{Ra}$ range $(2600 \leqslant \mathrm{Ra} \leqslant 3400)$. In order to distinguish between roll structures with opposite sense of rotation, we now plot the velocity $u_{1}$ (along $x$ ) at a representative point $\left(x_{1}=y_{1}=0.17419, z_{1}=0.2307\right)$ as a function of Ra. In these diagrams, the stable solutions are given as solid lines whereas the unstable solutions are given as dashed lines.

When the cavity is tilted, there is no motionless diffusive solution and the convection sets in as soon as $\mathrm{Ra}$ is different from zero. Due to the inclination $\theta$ chosen (around the $-z$
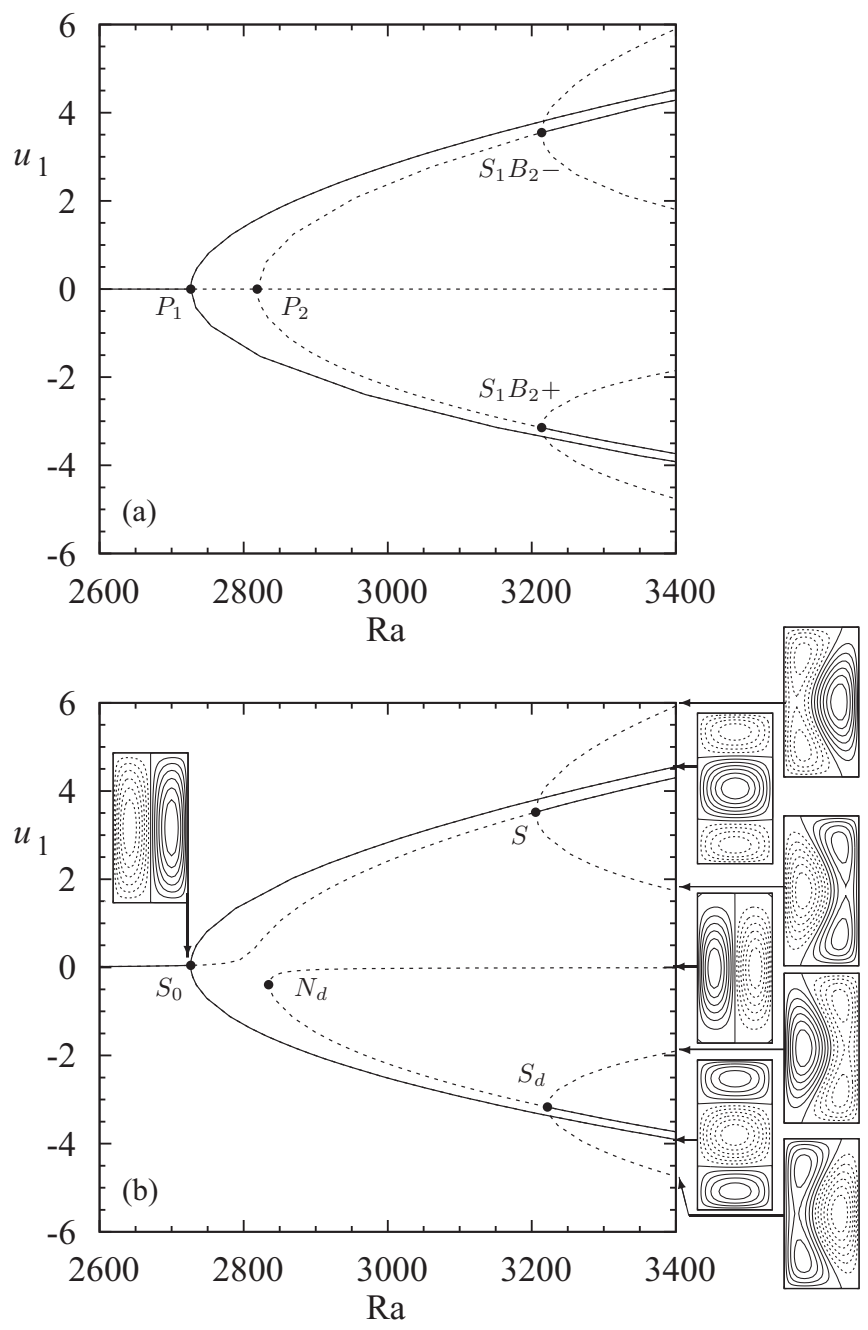

FIG. 4. Bifurcation diagram for the convection in a $1 \times 1 \times 2$ cavity in the case without inclination (a) and in the case with a small inclination $\left(\theta=0.01^{\circ}\right)(b)$, for the range $2600 \leqslant \mathrm{Ra} \leqslant 3400$. The abscissa indicates the Rayleigh number $\mathrm{Ra}$ and the ordinate the velocity $u_{1}$ (along $x$ ) at a representative point inside the cavity $\left(x_{1}=y_{1}=0.17419, z_{1}=0.2307\right)$. The stable solutions are given as solid lines. Solid circles indicate steady bifurcation points. The second primary bifurcation $P_{2}$ in the horizontal case is changed to a saddle-node point $N_{d}$ when the cavity is tilted. Insets in (b) are contours of the $u$ velocity (along $x$ ) in the mid- $y z$ plane $(x=0)$ for different solutions at $\mathrm{Ra}=3400$ and at the first bifurcation point $S_{0}$ $(\mathrm{Ra}=2726.53)$.

axis), this convection at small $\mathrm{Ra}$ corresponds to a weak single longitudinal roll with the same sense of rotation as the inclination angle ( $L-$ solution), a solution which has the $S_{P_{x y}}$ and $S_{A_{z}}$ symmetries. The primary bifurcation point $P_{1}$ is replaced by a secondary bifurcation point denoted as $S_{0}$. $S_{0}$ is still a pitchfork, as the eigenvector (which is close to a transverse two-roll flow) breaks the $S_{A_{z}}$ symmetry of the longitudinal roll. The solutions on the branches emerging from $S_{0}$ have only the $S_{P_{x y}}$ symmetry and cannot then be exact $T$ solutions, but, for $\theta=0.01^{\circ}$, they still look similar to the transverse two-roll solutions obtained in the horizontal case, and they are stable. The bifurcation $P_{2}$, which leads from no-flow to longitudinal roll solutions for $\theta=0^{\circ}$, is suppressed 


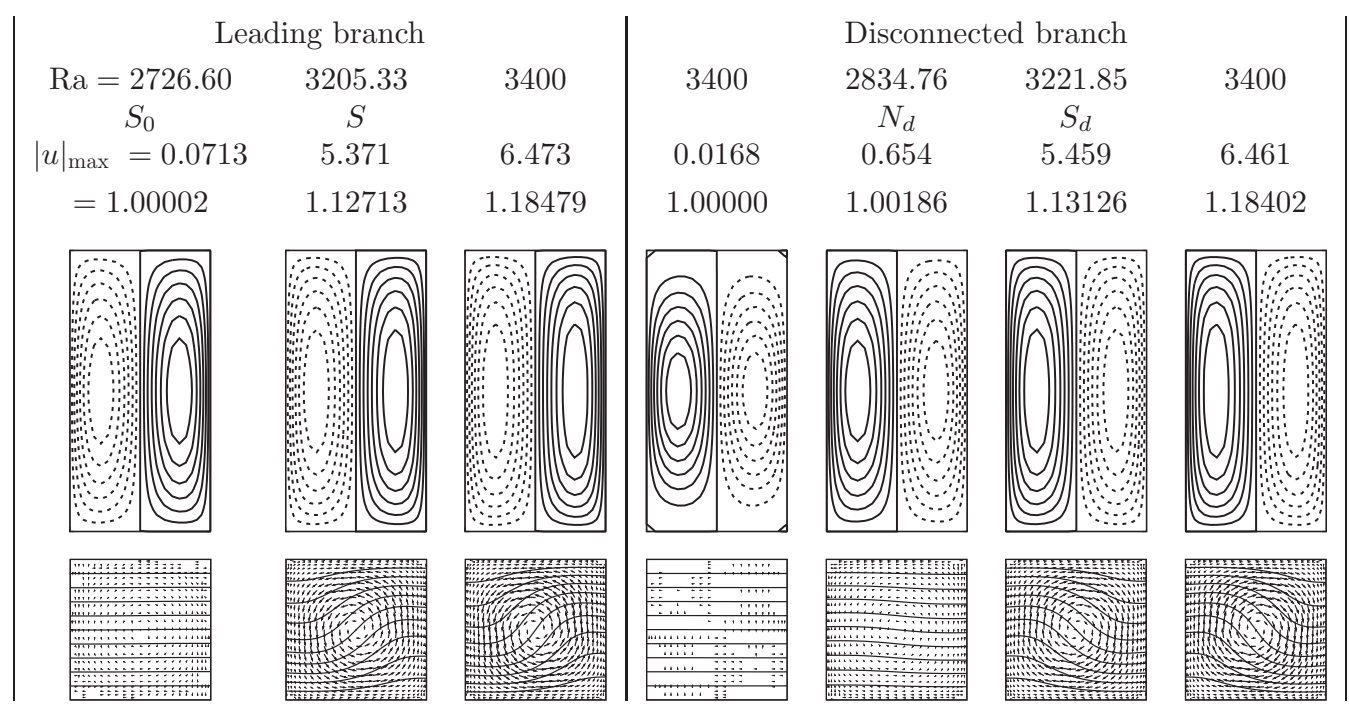

FIG. 5. Solutions corresponding to a single longitudinal roll, along the leading branch ( $L-$ solutions, left) and along the disconnected branch $\left(L+\right.$ solutions, right), in a $1 \times 1 \times 2$ cavity inclined by $\theta=0.01^{\circ}$. The leading branch is stable below $S_{0}$ and above $S$, the disconnected branch is stable above $S_{d}$. Contours of the $u$ velocity (along $x$ ) in the mid-yz plane $(x=0)$ in the upper pictures; temperature contours and velocity vector field in the mid- $x y$ plane $($ at $z=0$ ) in the lower pictures.

by the inclination (the solution at small $\mathrm{Ra}$ is already of this type) and is replaced by an imperfect bifurcation. We have a leading branch which is the continuity of the branch obtained at small Ra and corresponds to $L-$ solutions, and a disconnected branch which corresponds to $L+$ solutions (longitudinal roll with a sense of rotation opposite to the inclination angle) and exists beyond a saddle-node point $N_{d}$. Some solutions on the leading branch and on the disconnected branch are shown in Fig. 5. For the $L-$ solutions on the leading branch, the flow is still very weak at $S_{0}$ and associated with almost undeformed isotherms and a Nusselt number $\mathrm{Nu}$ very close to 1 . The flow increases for larger Ra values, and at $S$ the longitudinal roll is already quite intense and associated with clearly deformed isotherms in the $x y$ plane and Nusselt numbers larger than 1 . For the $L+$ solutions on the disconnected branch, the flow is very weak on the part of the branch which is close to the diffusive solution found in the horizontal case $(\mathrm{Nu} \approx 1)$. It is still weak, but increases, at the level of $N_{d}$ and becomes quite intense on the second part of the branch. As the flow for the $L+$ solutions is opposite to the flow for the $L-$ solutions, the deformation of the isotherms is also inverted and opposite to the inclination. The secondary bifurcations $S_{1} B_{2}+$ and $S_{1} B_{2}-$, which were found in the horizontal case, are still present for $\theta=0.01^{\circ}$, but they do not occur at the same critical value of $\mathrm{Ra}$ and they will evolve differently when increasing $\theta$. In the inclined case, these secondary bifurcations are denoted as $S$ for the leading branch and $S_{d}$ for the disconnected branch. They are still pitchfork as they break the $S_{A_{z}}$ symmetry of the $L+$ and $L-$ solutions. The leading branch, which was destabilized at $S_{0}$, is restabilized at $S$, whereas the disconnected branch is stabilized beyond $S_{d}$. The solutions obtained on the branches bifurcating at $S$ and $S_{d}$ correspond, as for $\theta=0^{\circ}$, to a kind of two-oblique-roll structure with the $S_{P_{x y}}$ symmetry. These solutions are all one time unstable. The critical values for the different bifurcation points just mentioned for $\theta=0^{\circ}$ and $0.01^{\circ}$ are given in Table VI.
The bifurcation diagram obtained for $\theta=0.01^{\circ}$ has clearly shown the four bifurcation points that are important in our inclined cavity situation, i.e., $S_{0}, N_{d}, S$, and $S_{d}$. It is then interesting to follow these points (expressed through their critical Rayleigh number) as a function of the inclination angle $\theta$ in order to provide a more complete description of the influence of the tilt on the stability of the system. The paths of these points for $0^{\circ} \leqslant \theta \leqslant 1^{\circ}$ are shown in Fig. 6 . The bifurcation points $S_{0}$ and $S$, which belongs to the same leading branch of $L-$ solutions, evolve differently with $\theta$ : $\mathrm{Ra}_{S_{0}}$ increases whereas $\mathrm{Ra}_{S}$ decreases. The two points quickly collide and disappear at a critical angle $\theta_{c} \approx 0.2714^{\circ}$. Beyond this value of $\theta$, due to the disappearance of its unstable portion between $S_{0}$ and $S$, the leading branch of $L-$ solutions becomes continuously stable. This change can be observed in Fig. 7 where bifurcation diagrams, focused on the leading branch dynamics, are given for $\theta=0.27^{\circ}$ and $0.28^{\circ}$, values below and above the critical angle $\theta_{c}$. This figure also shows that, in the same process at $\theta_{c} \approx 0.2714^{\circ}$, the two secondary branches originally initiated at $S_{0}$ and $S$ connect between them, giving origin to two disconnected branches. These disconnected branches, which have the same dynamics, are initiated at saddle-node points denoted as $N+$ and $N-$. The two parts of these branches corresponding to solutions closer to a transverse two-roll structure are stable whereas the two other parts are one time unstable. The saddle-node points $N+$ and $N-$ are in fact not created at $\theta_{c}$ but a little before, more

TABLE VI. Critical Rayleigh number $\mathrm{Ra}_{c}$ for the main bifurcation points found in the case of the horizontal cavity $\left(\theta=0^{\circ}\right)$ and the slightly inclined cavity $\left(\theta=0.01^{\circ}\right)$.

\begin{tabular}{lcccc}
\hline \hline$\theta$ & $P_{1} \rightarrow S_{0}$ & $P_{2} \rightarrow N_{d}$ & $S_{1} B_{2}-\rightarrow S$ & $S_{1} B_{2}+\rightarrow S_{d}$ \\
\hline $0^{\circ}$ & 2726.53 & 2818.78 & 3213.62 & 3213.62 \\
$0.01^{\circ}$ & 2726.60 & 2834.76 & 3205.33 & 3221.85 \\
\hline \hline
\end{tabular}




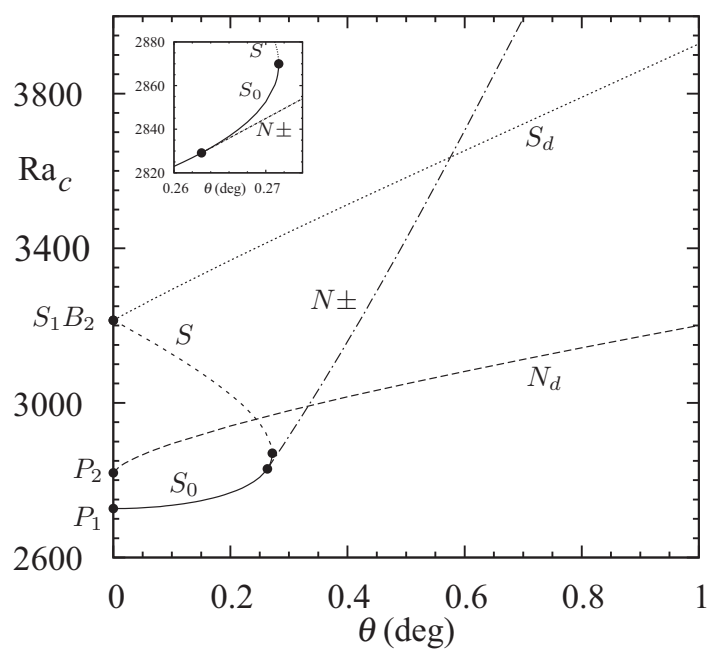

FIG. 6. Path of the different bifurcation points (secondary bifurcation points $S, S_{0}$, and $S_{d}$, saddle-node points $N_{d}$ and $N \pm$ ) as a function of $\theta\left(0^{\circ} \leqslant \theta \leqslant 1^{\circ}\right)$ for the tilted $1 \times 1 \times 2$ cavity. The abscissa is the inclination angle $\theta$ in degrees, and the ordinate is the critical Rayleigh number $\mathrm{Ra}_{c}$. Solid circles indicate specific bifurcation points: those at $\theta=0^{\circ}$, the connection point between $N \pm$ and $S_{0}$, and the collision point between $S_{0}$ and $S$. A zoom of the connection between $N \pm$ and $S_{0}$ is given in inset. The appearance of the two saddle-node points $N \pm$ from $S_{0}$ occurs at $\theta_{s} \approx 0.263^{\circ}$ before the collision between $S_{0}$ and $S$ at $\theta_{c} \approx 0.2714^{\circ}$.

precisely at $\theta_{s} \approx 0.263^{\circ}$ when the bifurcation at $S_{0}$ becomes slightly subcritical. The subcritical character of the bifurcation at $S_{0}$ can be seen in Fig. 7(a) for $\theta=0.27^{\circ}$. The saddle-node points $N+$ and $N-$ correspond to the same critical Rayleigh number and are globally denoted as $N \pm$. Their evolution with $\theta$ is depicted in Fig. 6: we see that their critical Rayleigh number $\mathrm{Ra}_{N \pm}$ strongly increases with $\theta$. The appearance of these saddle-node points at $\theta_{s} \approx 0.263^{\circ}$, just before $\theta_{c}$, is also shown in the inset of the same figure. We now consider the variation with $\theta$ of the two bifurcation points $N_{d}$ and $S_{d}$ belonging to the disconnected branch of $L+$ solutions (Fig. 6). Their critical Rayleigh numbers $\mathrm{Ra}_{N_{d}}$ and $\mathrm{Ra}_{S_{d}}$ both increase with $\theta$, but a little more slowly for $\operatorname{Ra}_{N_{d}}$ so that these points will not collide. The variation of $\mathrm{Ra}_{S_{d}}$ is particularly important as it gives the critical value of the Rayleigh number above which the $L+$ solutions are stable and can thus be physically observed. Note finally that the secondary bifurcation points $S$ and $S_{d}$, which correspond to the same critical Rayleigh number for $\theta=0^{\circ}$, have very different evolutions when $\theta$ is increased.

The variation with $\theta$ of the main bifurcation points, in a larger range of $\theta$ values $\left(0^{\circ} \leqslant \theta \leqslant 20^{\circ}\right)$, is depicted in Fig. 8 . The critical Rayleigh number for the different points $N \pm, S_{d}$, and $N_{d}$ increases with $\theta$. The strongest increase is found for $N \pm$, a smaller increase is observed for $S_{d}$, and the weakest increase is found for $N_{d}$. Again, the evolutions of $N \pm$ and $S_{d}$ are of particular importance because these points indicate the values of $\operatorname{Ra}$ and $\theta$ at which solutions are stabilized. For these different curves, there are limiting values of $\theta$ corresponding to a very strong increase of the critical Rayleigh number. These values are $\theta \approx 9.235^{\circ}, 9.445^{\circ}$, and $19.56^{\circ}$ for $N \pm, S_{d}$, and $N_{d}$, respectively. Beyond these limiting angles, it can be expected that the corresponding bifurcation point will disappear. For
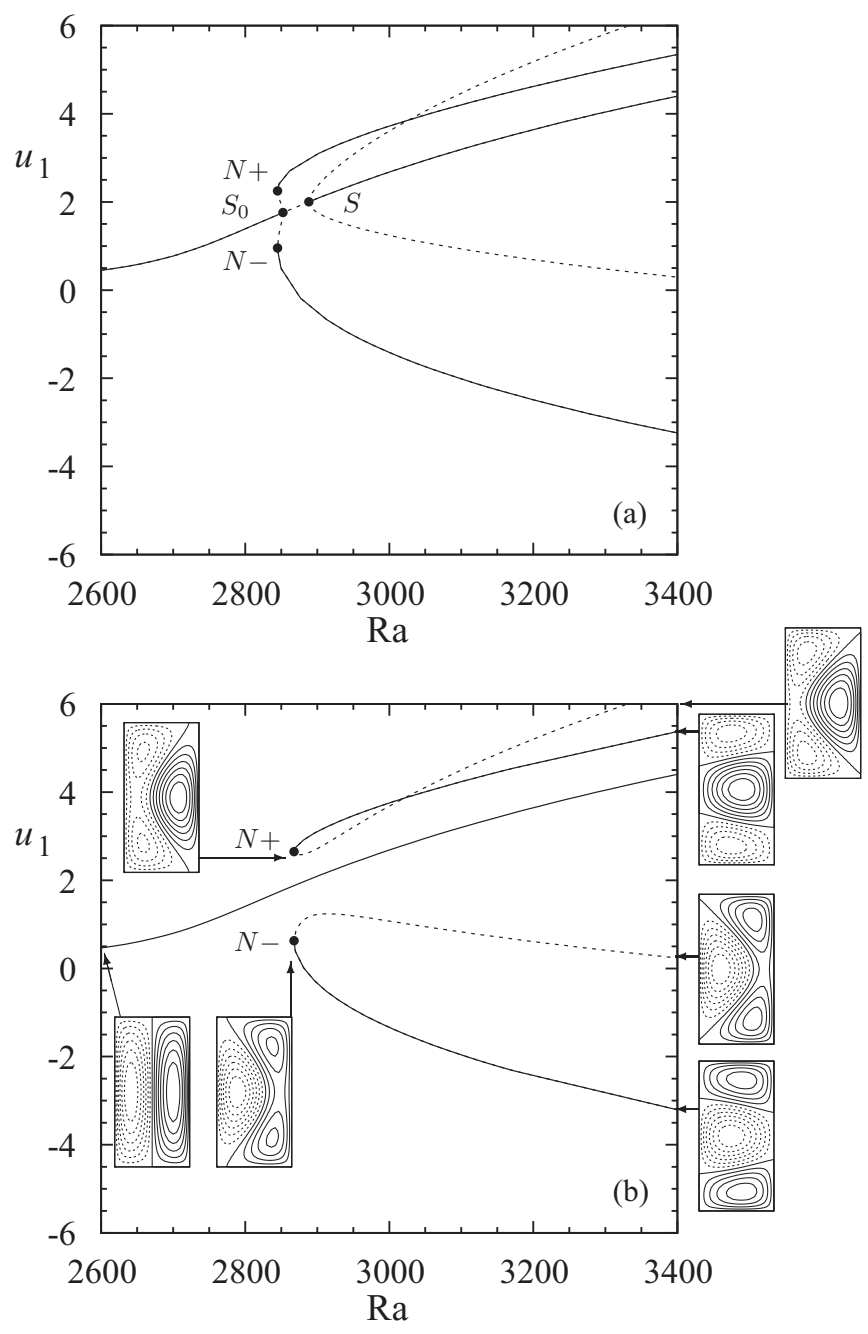

FIG. 7. Bifurcation diagrams in cases with inclination at $\theta=$ $0.27^{\circ}$ (a) and $\theta=0.28^{\circ}$ (b). Only what occurs in connection with the leading branch is shown. At $\theta=0.27^{\circ}$, a small Ra range on the leading branch, between $S_{0}$ and $S$, is still unstable, and the saddle-nodes $N \pm$ have already appeared on the secondary branches initiated at $S_{0}$. At $\theta=0.28^{\circ}$, just after the collision between $S_{0}$ and $S$, the leading branch is completely stable, and the secondary branches formerly initiated at $S_{0}$ and $S$ appear as two disconnected branches. Insets are contours of the $u$ velocity (along $x$ ) in the mid- $y z$ plane $(x=0)$ for different solutions, one solution on the leading branch at $\mathrm{Ra}=2600$, the solutions at the two saddle-node points $N+$ and $N-$ $(\mathrm{Ra}=2867.66)$, and the four solutions on the disconnected branches at $\mathrm{Ra}=3400$.

$N \pm$, the limiting value of $\theta$ is not shown in Fig. 8 because the critical Rayleigh numbers reached at this limiting value are about $\mathrm{Ra}_{c} \approx 540000$, i.e., far above the $\mathrm{Ra}$ values plotted in the graph. The solutions which are stabilized at $S_{d}$ and $N \pm$ are given as insets in Fig. 8. The solution obtained at $S_{d}$, which corresponds to the longitudinal roll $L+$ solution, is shown for $\theta=7^{\circ}$. At $N \pm$, we have two equivalent solutions which are stabilized and look like two-oblique-roll structures. One of them is shown for $\theta=4^{\circ}$ in Fig. 8. The other solution can be deduced by applying the $S_{A_{z}}$ symmetry. They will be denoted as $O \pm$ solutions in the following. 


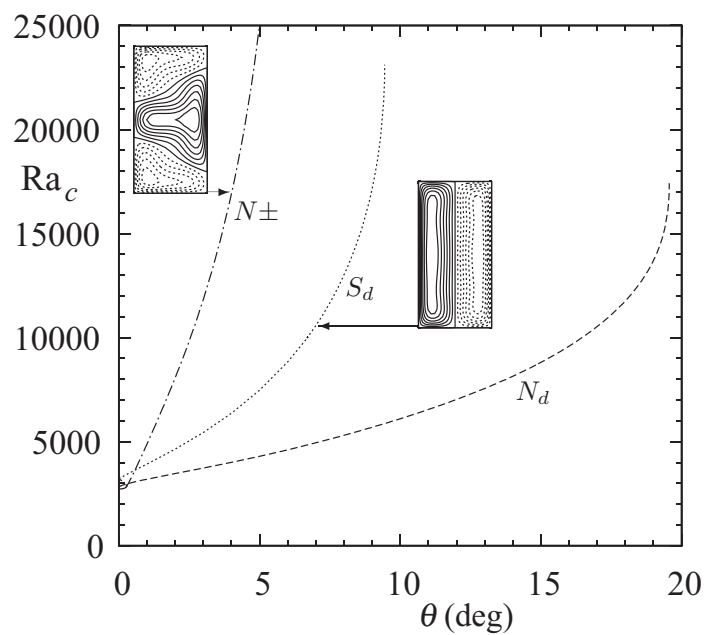

FIG. 8. Path of the different bifurcation points (secondary bifurcation point $S_{d}$, saddle-node points $N_{d}$ and $N \pm$ ) as a function of $\theta\left(0^{\circ} \leqslant \theta \leqslant 20^{\circ}\right)$ for the tilted $1 \times 1 \times 2$ cavity. For the different bifurcations, there are limiting values of $\theta$ corresponding to a very strong increase of the critical Rayleigh number. These values are $\theta \approx 9.235^{\circ}, 9.445^{\circ}$, and $19.56^{\circ}$ for $N \pm, S_{d}$, and $N_{d}$, respectively. For $N \pm$, the $\mathrm{Ra}_{c}$ values reached at the limiting value of $\theta$ are about 540000 , i.e., far above the Ra values plotted in the graph. Insets are contours of the $u$ velocity (along $x)$ in the mid- $y z$ plane $(x=0)$ for solutions which are stabilized at $S_{d}\left(\mathrm{Ra}_{c}=10584.24\right)$ and $N \pm$ $\left(\mathrm{Ra}_{c}=16919.44\right)$ for $\theta=7^{\circ}$ and $4^{\circ}$, respectively.

We finally present the domains of existence of the three different stable solutions $L-, L+$, and $O \pm$ in the Ra- $\theta$ parameter space in Fig. 9. Two plots are given, showing different ranges of $\theta$ and $\mathrm{Ra}$ values: for $\theta \leqslant 1^{\circ}$ where interesting dynamics between the solutions are observable, and for $0^{\circ} \leqslant \theta \leqslant 20^{\circ}$ where a description for a broader inclination range is shown, from which the results in the whole $\theta$ range $\left(0^{\circ} \leqslant \theta \leqslant 90^{\circ}\right)$ can be deduced. From the different domains of existence determined for the $L-, L+$, and $O \pm$ stable solutions, we can also define zones in the $\mathrm{Ra}-\theta$ parameter space where one or several stable solutions are expected to exist. Five different zones can thus be found, which are shown in Fig. 9 and labeled with an encircled number.

We first comment on the domains of existence for each stable solution. The leading longitudinal roll $L-$ solution (domain with grey background, zones $1,3,4,5$ ) exists in the whole $\mathrm{Ra}-\theta$ range studied, except in the small area with a white background below $\theta_{c} \approx 0.2714^{\circ}$, and for $\theta=0^{\circ}$ below $\operatorname{Ra}_{S_{1} B_{2}}=3213.62$. This $L-$ solution indeed also exists for larger values of $\theta$ (up to $\theta=90^{\circ}$ ), and it is in this domain the only existent solution (see the solutions obtained for $\mathrm{Ra}=$ 10000 and increasing $\theta$ in Fig. 10). The opposite longitudinal roll $L+$ solution (domain with up-left directed oblique lines, zones 4,5 ) exists above the line corresponding to the path of the $S_{d}$ bifurcation point (secondary bifurcation point on the disconnected branch). This line begins at $\operatorname{Ra}_{S_{1} B_{2}}=3213.62$ for $\theta=0^{\circ}$, increases almost linearly with $\theta$, before a very steep increase for $\theta \approx 9.445^{\circ}$. This value of $\theta$ seems to be the limit to get such a $L+$ solution. Finally, the two two-oblique-roll $O \pm$ solutions (domain with up-right directed oblique lines, zones 2, 3, 5) exist above the line corresponding to the
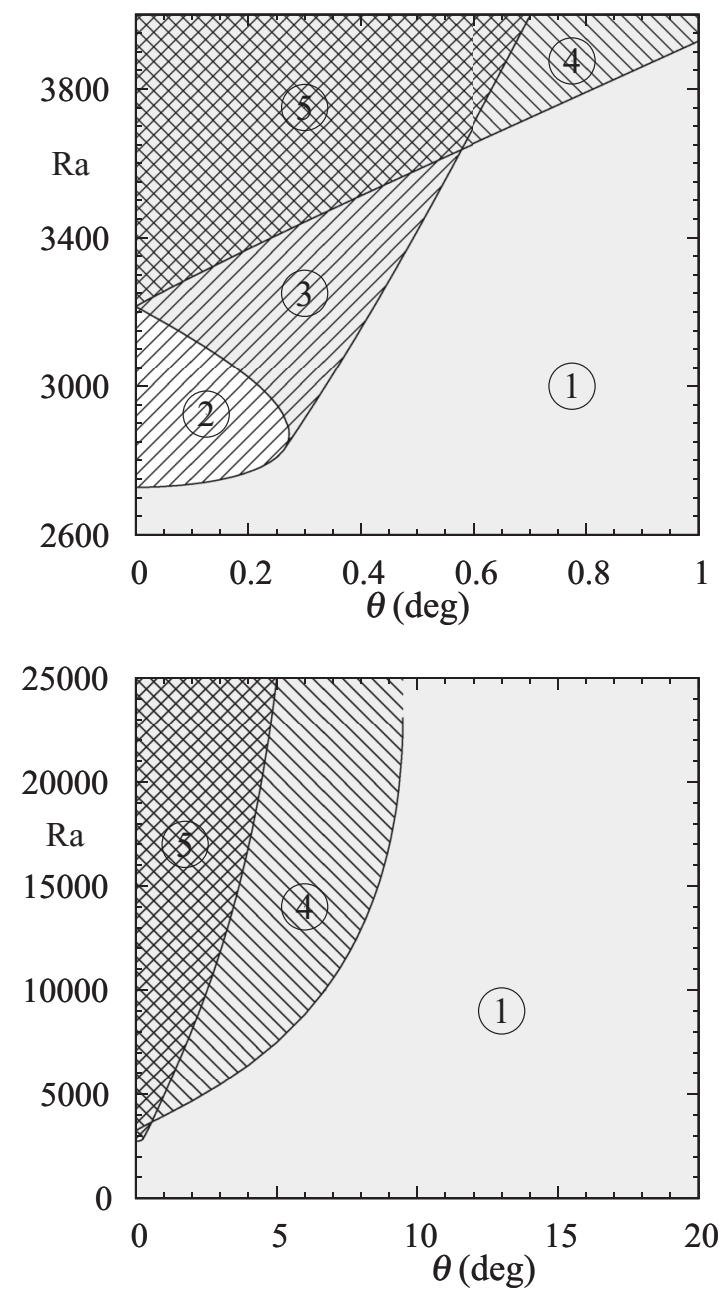

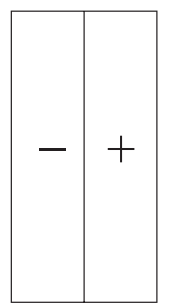

$L-$

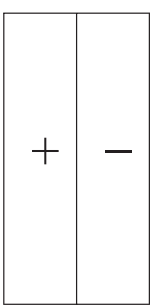

$L+$

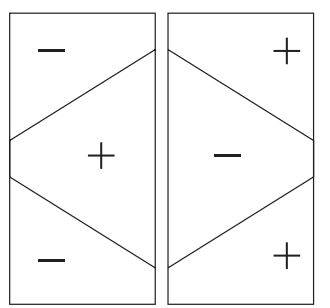

$O \pm$
FIG. 9. Domains of existence of the different stable solutions in the $\operatorname{Ra}-\theta$ parameter space: the leading longitudinal roll $L-$ solution (domain with grey background), the opposite longitudinal roll $L+$ solution (domain with up-left directed oblique lines), the two two-oblique-roll $O \pm$ solutions (domain with up-right directed oblique lines). We can thus define five zones in the $\operatorname{Ra}-\theta$ parameter space (labeled with an encircled number) where one or several stable solutions exist. We have one type of solutions in zones 1 and 2, two types in zones 3 and 4 , and three types in zone 5 . The first plot is a zoom of the second plot for small values of $\theta$. Note that the leading longitudinal roll $L-$ solution (domain with grey background and zone 1) indeed exists up to $\theta=90^{\circ}$. A sketch of the different solutions, showing the sign of the $u$ velocity (along $x$ ) in the mid- $y z$ plane $(x=0)$, is finally given.

successive paths of the $S_{0}$ and $N \pm$ bifurcation points (the first bifurcation point on the leading branch and the saddle-node 

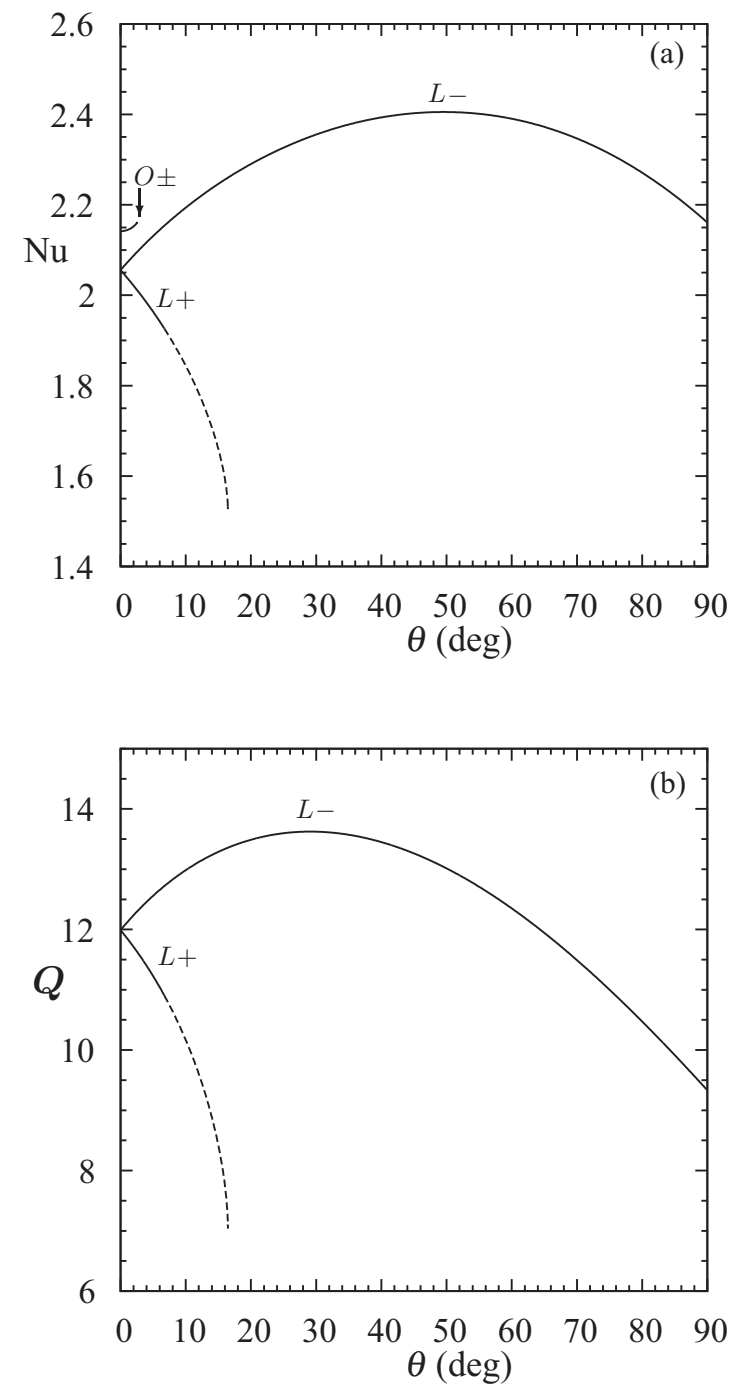

FIG. 10. Nusselt number $\mathrm{Nu}$ (a) and dimensionless flow rate $Q$ (b) as a function of the inclination $\theta$ for the stable $L-$ and $L+$ solutions at $\mathrm{Ra}=10000$ (solid lines). $\mathrm{Nu}$ and $Q$ are also given for the unstable $L+$ solutions as dashed lines. The Nusselt number is also given for the stable $O \pm$ solution in (a). As expected from Fig. 9, the $O \pm$ solution exists and is stable in a small range of $\theta$ values, up to the saddle-node point $N \pm$ at $\theta=2.5418^{\circ}$. The $L+$ solution exists up to the saddle-node point $N_{d}$ at $\theta=16.4577^{\circ}$, but is stable only up to the secondary bifurcation point $S_{d}$ at $\theta=6.697^{\circ}$. The $L-$ solution is stable in the whole range of $\theta$ values, up to $\theta=90^{\circ}$. The Nusselt number for this $L-$ solution is maximum for $\theta \approx 49.55^{\circ}$, whereas the maximum for $Q$ occurs for $\theta \approx 29.1^{\circ}$.

points which are further generated, respectively). This line begins at $\mathrm{Ra}_{P_{1}}=2726.53$, follows the curve of $S_{0}$, and then increases strongly along the path of $N \pm$, so that it intersects the $S_{d}$ path. The increase is almost linear for small values of $\theta$, but becomes then steeper. The value $\theta \approx 5^{\circ}$ reached for $\mathrm{Ra}_{c}=25000$ (limit of the graph) is not the limit value for the $N \pm$ path. As indicated before, the limit value was obtained at $\theta \approx 9.235^{\circ}$ and corresponded to $\mathrm{Ra}_{c} \approx 540000$. We do not know if stable $O \pm$ solutions can be obtained up to such large values of $\mathrm{Ra}_{c}$. In any case, as the destabilization of the stable $B_{1}$ and $B_{2}$ branches (from which the different $L-, L+$, and
$O \pm$ stable solutions are obtained when $\theta \neq 0^{\circ}$ ) only occurs beyond $\mathrm{Ra}=80000$ (see at the end of Sec. IIIC), we can expect that the domains of existence of the different solutions will extend far above the limit value $\mathrm{Ra}=25000$ of our plots. We finally describe the five zones in the Ra- $\theta$ parameter space where one or several stable solutions exist. In zones 1 and 2, we only have one type of stable solution: the $L-$ solution in zone 1 (remember that this zone 1 extends up to $\theta=90^{\circ}$ ) and the $O \pm$ solutions in zone 2 . In zones 3 and 4 , we have two types of stable solutions: the $L-$ solution together with the $O \pm$ solutions in zone 3 , and the $L$ - solution together with the $L+$ solution in zone 4 . Finally in zone 5 , the three types of stable solutions can be obtained. We see that zones 2 and 3 are very small, whereas the other zones have a far larger extent, particularly zone 1 .

All these stable solutions, however, cannot be reached so easily. It is then interesting to give some comments on the possibility or difficulty to reach them. In the horizontal situation, the transverse roll $T$ solution, which is triggered at the first primary bifurcation $P_{1}$, is naturally obtained from the no-flow solution by increasing Ra. This $T$ solution will also be eventually reached by increasing $\mathrm{Ra}$ when $\theta$ is nonzero but smaller than $\theta_{s} \approx 0.2714^{\circ}$. In contrast, if $\theta$ is larger than $\theta_{s}$, only the longitudinal roll $L-$ solution will be obtained if gradually increasing $\mathrm{Ra}$ from $\mathrm{Ra}=0$. This indicates that a good horizontality of the cavity $\left(\theta<\theta_{s}\right)$ is required to obtain the expected $T$ solution in the Rayleigh-Bénard situation. If we now proceed by increasing $\theta$ at constant $\mathrm{Ra}$, we can first state that for $\mathrm{Ra} \leqslant \mathrm{Ra}_{P_{1}}(\mathrm{Ra} \leqslant 2726.53)$, the $L-$ solution will be continuously obtained up to $\theta=90^{\circ}$. Beyond $\operatorname{Ra}_{P_{1}}$, the transverse roll $T$ solution obtained at $\theta=0^{\circ}$ will be maintained, although transformed into an oblique roll $O \pm$ solution, up to the limit $\theta$ values corresponding to the $S_{0}$ and $N \pm$ curves shown in Figs. 6 and 8. Beyond these limit $\theta$ values (which are in the range $0 \leqslant \theta \leqslant 9.235$ ), we can expect that the $L-$ solution will still be obtained. In contrast, for decreasing $\theta$ (from, for example, $\theta=90^{\circ}$ ) at constant Ra, the $L$ - solution will be always obtained, except for $2726.53 \leqslant \mathrm{Ra} \leqslant 3213.62$, in the small domain close to $\theta=0^{\circ}$ corresponding to the zone 2 in Fig. 9, where an $O \pm$ solution can be expected. Finally, the $L+$ solution, though it is stable in a certain parameter range, seems difficult to obtain as it is on a disconnected branch. One possibility would be to get an $L-$ solution at small $\theta$ in this stable parameter range and to invert the tilt of the cavity (opposite value of $\theta$ ). We can expect that this solution, which will be an $L+$ solution due to the tilt inversion, will proceed and can be maintained for increased $\theta$ values up to the limit $\theta$ values corresponding to the $S_{d}$ curve shown in Figs. 6 and 8.

\section{E. Discussion}

It is interesting to compare our results to those obtained in some papers dealing with the influence of a tilt on RayleighBénard convection. We will consider the papers of Cliffe and Winters [16], Adachi [19], and Ozoe et al. [18].

Cliffe and Winters [16] consider the Rayleigh-Bénard convection in a two-dimensional square cavity tilted by an angle $\theta$ with the horizontal. This two-dimensional cavity can also be seen as a square duct with an infinite extension, to be compared with our three-dimensional finite length square duct. 
The sidewalls are insulated as in our case. The authors show that the pitchfork bifurcation at which the one-roll solutions are triggered for $\theta=0^{\circ}$ is changed by a cusp catastrophe when $\theta$ is not zero: the flow then develops smoothly from zero Rayleigh number on a leading branch, and two other solutions appear on a disconnected branch terminating at a saddle-node point, one of these solutions being stable. The stable solution on the disconnected branch is called anomalous and it corresponds to a one-roll flow with opposite sense of rotation with regard to the stable leading solution. It is exactly what we obtain in our three-dimensional situation at the primary bifurcation point $P_{2}$ where a longitudinal one-roll solution is triggered at $\theta=0^{\circ}$ : we see the disappearance of $P_{2}$ when a tilt is imposed and the presence of a leading branch and a disconnected branch. The difference with the two-dimensional case is that in the three-dimensional situation $P_{2}$ is not the first primary bifurcation point to appear, so that the branches initiated at $P_{2}$ for $\theta=0^{\circ}$ and those obtained from them when $\theta$ is not zero are all unstable (at least for small $\theta$ and close to $P_{2}$ ). Cliffe and Winters [16] also give the path of the saddle-node point in the $\operatorname{Ra}_{c}-\theta$ parameter space and mention the cusp shape close to $\theta=0^{\circ}$ and the limiting value for this path at $\theta=22^{\circ}$. The cusp shape can be seen in our case in Fig. 6 $\left(N_{d}\right.$ curve close to $P_{2}$ ) and the limiting value is shown in Fig. 8 and corresponds to $\theta \approx 19.56^{\circ}$. The limiting value is about $2^{\circ}$ smaller in our three-dimensional situation than in the two-dimensional cavity, but both values are roughly about $20^{\circ}$. The three-dimensional confinement may be responsible for the difference in the limiting angle.

Adachi [19] considers the stability of natural convection in an inclined square duct with perfectly conducting sidewalls. His stability analysis considers two-dimensional basic flows in the square cross section, which could be a conducting solution or a one-roll solution, and the perturbations are three dimensional. Without inclination, in this square duct with infinite extension, the first transition from the no-flow conducting solution is to a transverse multiroll solution denoted as the $T$-roll solution and occurs at $\mathrm{Ra}_{c}=2936.4$. The transition to a longitudinal one-roll solution, denoted as the $L$-roll solution, occurs later at $\mathrm{Ra}_{c}=5011.7$. These thresholds can be compared to our thresholds $\mathrm{Ra}_{P_{1}}=2726.53$ and $\mathrm{Ra}_{P_{2}}=2818.78$ leading to $T$ and $L$ solutions, respectively. In both cases, the thresholds for the $T$ solutions occur before those for the $L$ solutions. Our values are however smaller. Two effects influence the thresholds: the three-dimensional confinement must increase the thresholds, but the adiabatic boundaries must decrease them. The second effect seems to overcome the first effect in the situation we have chosen. At $\theta=0^{\circ}$, the $L$-roll solution is found by Adachi [19] to be unstable, which is expected because of the previous transition to $T$ rolls, but the further stabilization of this $L$-roll solution is not mentioned. Concerning the $T$ rolls (which are three-dimensional structures), his method is not able to calculate them. Adachi [19] further considers a small inclination, $\theta=0.01^{\circ}$. For the $L$-roll solution, he is able to calculate the leading branch, and the disconnected branch, which is initiated at a saddle-node point at $\mathrm{Ra}_{c}=5042$. The stability analysis of these branches shows that the leading branch is stable up to $\mathrm{Ra}_{c}=2941.6$, which is the new threshold associated to $T$-roll perturbations for $\theta=0.01^{\circ}$, whereas the disconnected branch is unstable. The same type of behavior has been found in our case. He also indicates that the transition at $\mathrm{Ra}_{c}=2941.6$ must lead to a superposed flow between $L$ roll and $T$ rolls, which corresponds to our oblique roll $O \pm$ solutions. Our three-dimensional calculations in a truncated square duct of dimensionless length 2 have then confirmed the findings of Adachi [19], but for adiabatic sidewalls. Furthermore, our calculations have also extended the results to all the three-dimensional solutions, which were followed by continuation, and in a large range of $\theta$ values. Our results have also put into light new behaviors as the further stabilization of the $L$-roll branch, which lead to very interesting dynamics when the cavity is tilted. The dynamics are particularly rich for small angles of inclination (e.g., $\theta \leqslant 1^{\circ}$ at $\operatorname{Pr}=1$ ). Such slight inclination angles could be of great relevance in real physical situations where the parallelepiped cavity is intended to be horizontal but no specific precautions are taken to increase the accuracy of its horizontality.

Ozoe et al. [18] study the heat transfer properties of natural convection in an inclined square channel with insulated sidewalls. They perform two-dimensional simulations in the inclined square cross section for a channel considered as infinite and obtain one-roll solutions featuring the longitudinal roll solutions obtained in the three-dimensional channels. They also perform experiments with glycerol in a threedimensional convection channel with dimensions $15 \mathrm{~mm} \times$ $15 \mathrm{~mm} \times 270 \mathrm{~mm}$, i.e., with an aspect ratio $A_{z}=18$. In these experiments, the inclination $\theta$ is progressively decreased from $90^{\circ}$ to $0^{\circ}$. For fixed values of the Rayleigh number, they numerically and experimentally obtain the Nusselt number as a function of the inclination angle $\theta$. The numerical and experimental curves agree quite well for $\theta>10^{\circ}$ and indicate a maximum heat transfer for $\theta \approx 50^{\circ}$. The flow in this $\theta$ range corresponds to a longitudinal roll. For smaller inclinations, transverse rolls or more complex flow structures (which are not precisely described) are obtained experimentally (whereas only longitudinal rolls can be obtained numerically), inducing the presence of a minimum heat transfer at an inclination of about $10^{\circ}$. It is also indicated that the transverse roll solution is the solution obtained when the cavity is kept almost horizontal, with say less than one degree of inclination. Interesting comparisons with our results can be obtained. The longitudinal roll mentioned by Ozoe et al. [18] for $\theta>10^{\circ}$ must be the longitudinal roll $L-$ solution obtained in our study. From Fig. 9, this $L-$ solution is also in our case the only solution for $\theta>10^{\circ}$. The more complex solutions obtained for $\theta<10^{\circ}$ could be our $O \pm$ solutions. For small inclination angles, these $O \pm$ solutions look like transverse roll solutions when $\mathrm{Ra}$ is sufficiently high [see the flow structures in Fig. 7(b) for $\theta=0.28^{\circ}$ and $\mathrm{Ra}=3400$ ] and, for $\theta \leqslant \theta_{c} \approx 0.2714^{\circ}$, these solutions can be reached easily by simply increasing $\mathrm{Ra}$ (in contrast, for larger inclinations, they belong to disconnected branches). This observation is in accordance with the fact that Ozoe et al. [18] mention the existence of transverse roll solutions only for $\theta \lesssim 1^{\circ}$. Concerning the $L+$ solutions, which, as the $L-$ solutions, are quite easily recognizable, we can think that Ozoe et al. [18] did not obtain them due to the fact that these solutions are on a disconnected branch and the authors gradually decreased the inclination of the cavity from $\theta=90^{\circ}$ step by step while 
fixing Ra. Ozoe et al. [18] also did not mention these $L+$ solutions in their two-dimensional numerical study. In any case, the comparisons cannot be expected to be perfect due to the differences between our approaches. First our simulations concern a finite-length square duct with $A_{z}=2$ compared to $A_{z}=18$ and $A_{z} \rightarrow \infty$ in the experiment and calculations of Ozoe et al. [18], respectively. Then, our calculations are performed for a Prandtl number $\operatorname{Pr}=1$ whereas Ozoe et al. [18] use $\operatorname{Pr}=10$ in their two-dimensional calculations and give values of Pr between 2690 and 2580 for their experiments. Finally, our simulations can determine all the possible stable solutions by continuation whereas Ozoe et al. [18] realize temporal evolutions (either numerically or experimentally) from different initial conditions and the stable solutions obtained are those which exert the strongest attraction in the corresponding phase space.

Nevertheless, in order to complement our comparisons with the work of Ozoe et al. [18], we have calculated the Nusselt number as a function of the inclination angle for the stable $L-, L+$, and $O \pm$ solutions obtained for $\mathrm{Ra}=10000$. The results are shown in Fig. 10(a) for $0^{\circ} \leqslant \theta \leqslant 90^{\circ}$. The values of the flow rate $Q$ for the $L-$ and $L+$ solutions are given in Fig. 10(b). This dimensionless flow rate has been obtained by integration of the $u$ velocity (along $x$ ) on one half of the $y z$ plane $(y \leqslant 0$ or $y \geqslant 0)$. For $\mathrm{Ra}=10000$, the $L-$ solution is stable in the whole range of studied $\theta$ values. The curve obtained in the $\mathrm{Nu}-\theta$ parameter space for this $L-$ solution is similar to the curves obtained by Ozoe et al. [18]. The Nusselt number is found to be maximum for $\theta \approx 49.55^{\circ}$, in close agreement with the value $\theta \approx 50^{\circ}$ given by Ozoe et al. [18]. The Nusselt number values obtained with this $L-$ solution at $\mathrm{Ra}=10000$ are within the range $2.05 \lesssim \mathrm{Nu} \lesssim 2.4$. Despite the very different Prandtl number, these values are a little smaller but not far from the values obtained by Ozoe et al. [18] (e.g., $2 \lesssim \mathrm{Nu} \lesssim 2.4$ for $\mathrm{Ra}=8000$ and $2.2 \lesssim \mathrm{Nu} \lesssim 2.65$ for
$\mathrm{Ra}=11000)$. For $\mathrm{Ra}=10000$, the $O \pm$ solutions exist and are stable in a small range of $\theta$ values, from $0^{\circ}$ up to the saddle-node point $N \pm$ at $\theta=2.5418^{\circ}$. The Nusselt number for these $O \pm$ solutions increases with $\theta$, and at fixed $\theta$, the values obtained are larger than those for the $L-$ solution. Concerning the $L+$ solution, it exists up to the saddle-node point $N_{d}$ at $\theta=16.4577^{\circ}$, but is stable only up to the secondary bifurcation point $S_{d}$ at $\theta=6.697^{\circ}$ (the unstable solutions are given as dashed lines in Fig. 10). The Nusselt number for this $L+$ solution strongly decreases when $\theta$ is increased, and this decrease becomes very steep, almost vertical, at the existence limit of the solution at the saddle-node point $N_{d}$. For these $L+$ solutions, the decrease of $\mathrm{Nu}$ is justified by the fact that the intensity of the roll, which rotates in a sense opposite to the inclination, is decreased when the inclination is increased [see in Fig. 10(b)]. Note that, as expected, the $L-$ and $L+$ solutions have the same Nusselt number at $\theta=0^{\circ}$. We can finally mention that, in our case, we do not obtain a minimum Nusselt number as the one found experimentally by Ozoe et al. [18] for $\theta \approx 10^{\circ}$. Concerning the flow rate $Q$ shown in Fig. 10(b), we see that its variation with $\theta$ for the $L-$ solution has a similar bell shape as the $\mathrm{Nu}$ curve, but with a maximum which occurs at a far smaller inclination, at $\theta \approx 29.1^{\circ}$. The differences which can be observed between the $\mathrm{Nu}$ and $Q$ curves indicate that, when $\theta$ is increased, the heat transfer is not directly correlated with the intensity of the flow for these $L-$ solutions.

In order to clarify these observations, the flow structures for these stable $L-$ solutions at $\mathrm{Ra}=10000$ and for different values of the inclination $\theta$ from $\theta=0^{\circ}$ to $\theta=90^{\circ}$ are depicted in Fig. 11. In each case, the velocity vector field and the isovalues of the temperature are plotted in the square cross section at $z=0$ (mid- $x y$ plane), which is the main flow plane for these $L-$ solutions. As expected, a one-roll flow is found for all these $L-$ solutions.

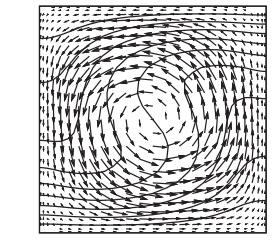

$\theta=0^{\circ}$

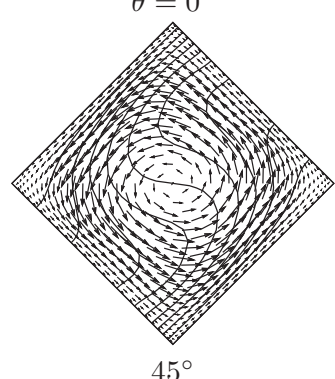

$45^{\circ}$

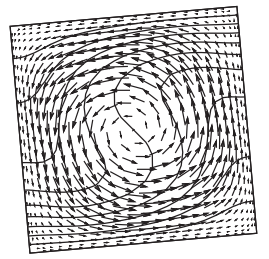

$5^{\circ}$

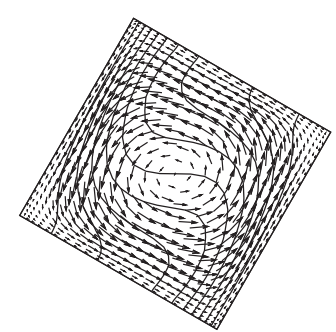

$60^{\circ}$

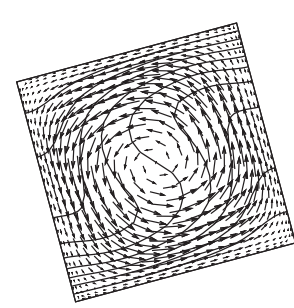

$15^{\circ}$

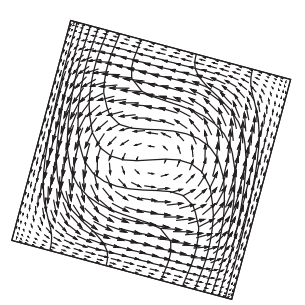

$75^{\circ}$

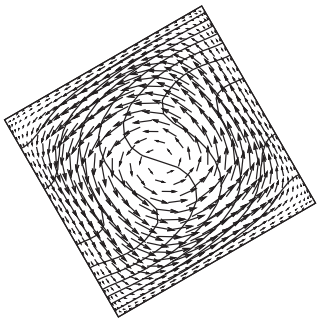

$30^{\circ}$

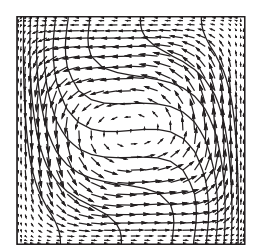

$90^{\circ}$

FIG. 11. Velocity vector field and isovalues of the temperature in the square cross section at $z=0$ (mid- $x y$ plane) for the stable $L-$ solutions at $\mathrm{Ra}=10000$ and for different values of the inclination $\theta$. The isotherms are rather horizontal near the boundaries and vertical in the core for $\theta=0^{\circ}$ and rather vertical near the boundaries and horizontal in the core for $\theta=90^{\circ}$. Correspondingly, the flow evolves from a circular roll at $\theta=0^{\circ}$ to a more square-shaped roll at $\theta=90^{\circ}$. 
To better analyze these results, we have to remember that the isotherms create a buoyancy effect only when they are not horizontal, the strongest effect being connected to vertical isotherms. We can observe that for $\theta=0^{\circ}$, a situation where the diffusive state corresponds to horizontal isotherms, the isotherms remain almost horizontal along the boundaries (in connection with the no-slip and adiabatic conditions at these boundaries) and are only deformed by the flow in the core of the cavity. The buoyancy effect is then mainly effective in the core of the cavity and the flow then rather appears as a circular roll. In this situation, the flow can be strong, without too strongly affecting the thermal boundary layers, i.e., the Nusselt number can be moderately affected by the intensity of the flow. In contrast, for $\theta=90^{\circ}$, a situation where the diffusive state would correspond to vertical isotherms, the isotherms remain rather vertical along the boundaries and become almost horizontal in the core. The buoyancy effect is then mainly effective along the boundaries and the flow appears as a more square-shaped roll with a quiescent core. In that case, the flow can be reduced by the absence of buoyancy effect in the core, but can nevertheless affect the thermal boundary layers and maintain a relatively strong Nusselt number. This can explain that the Nusselt number remains strong for $\theta=90^{\circ}$ while the intensity of the flow rate is reduced, whereas a smaller value of $\mathrm{Nu}$ is obtained for $\theta=0^{\circ}$ with a much stronger flow rate.

We can finally analyze the changes which occur when $\theta$ is increased from $\theta=0^{\circ}$. With the inclination of the cavity $(\theta \neq 0)$, the buoyancy effect becomes effective closer to the boundaries (inclination of the isotherms close to the boundaries), which induces an increase of the flow, but this buoyancy effect will be progressively reduced in the core where the isotherms evolve from vertical to horizontal, which will reduce the increase of the flow. According to Fig. 10, the increase of the flow rate is effective up to $\theta \approx 29.1^{\circ}$, and it induces an increase of $\mathrm{Nu}$. For larger values of $\theta$, as shown for $\theta \geqslant 45^{\circ}$ in Fig. 11, the isotherms become horizontal in the core (no buoyancy effect). The flow rate will decrease, but the Nusselt number will continue to increase due to the sustained flow along the boundaries. According to Fig. 10, $\mathrm{Nu}$ will eventually decrease only beyond $\theta \approx 49.55$, but this decrease will be less steep than the decrease of the flow rate.

\section{CONCLUDING REMARKS}

A continuation method developed from a three-dimensional spectral finite element code and particularly well suited for the studies involving complex bifurcation diagrams has been used to study the three-dimensional convection in a rectangular parallelepiped cavity tilted around its longest axis for a fluid with $\operatorname{Pr}=1$. The cavity has a square cross section and an aspect ratio $A_{z}=$ length/height $=2$ (finite-length square duct). When the cavity is horizontal (Rayleigh-Bénard situation), a precise bifurcation diagram has been obtained featuring all the different branches issued from the first primary bifurcations or at subsequent secondary bifurcations. Among these branches (which correspond to solutions with different symmetries), the branches of interest, however, are those initiated at the first two primary bifurcations $P_{1}$ and $P_{2}$ because of the existence of stable solutions on these two branches. In contrast, the branches and sub-branches initiated beyond $P_{2}$ were found unstable in the whole Ra range studied. The first branch initiated at $P_{1}$ corresponds to transverse rolls with axis perpendicular to the axis of the duct ( $T$ solution) and is stable right from its onset at $\mathrm{Ra}_{P_{1}}=2726.53$. The second branch initiated at $P_{2}$ corresponds to a longitudinal roll with its axis parallel to the axis of the duct ( $L$ solution) and is stabilized at a secondary bifurcation point $S_{1} B_{2}\left(\mathrm{Ra}_{P_{2}}=2818.78, \mathrm{Ra}_{S_{1} B_{2}}=\right.$ 3213.62).

The study has been extended from the Rayleigh-Bénard situation to the case where a tilt is imposed to the finite-length duct. This tilt, which is considered around the longest axis of the rectangular enclosure, can be a slight defect of the experimental device or can be imposed on purpose. Besides the fundamental interest of this study, our idea was also to complement the previous studies of Adachi [19] and Ozoe et al. [18] for more extended square ducts. In these situations, as in our case, without inclination the transition to transverse rolls occurs before the transition to the longitudinal roll, and the tilt (around an axis parallel to the axis of the duct) will promote a longitudinal roll (denoted as $L-$ solution in our study).

Our numerical investigation has yielded interesting results. As expected, the stable no-flow diffusive solution is replaced by a stable $L-$ solution in the inclined case. For small tilt angles $\theta$, the transition that occurred at $P_{1}$ for $\theta=0^{\circ}$ is maintained, so that there is a destabilization of the $L-$ solution (now at a secondary bifurcation called $S_{0}$ ) leading to stable perturbed $T$ solutions denoted as $O \pm$ solutions, which will become clear oblique roll solutions for larger values of $\theta$. In contrast, the bifurcation at $P_{2}$, which leads to $L$ solutions for $\theta=0^{\circ}$, is replaced by an imperfect bifurcation. Here, the leading branch of $L-$ solutions continuously evolves for increasing $\mathrm{Ra}$, with an increasing intensity of the flow, whereas there is a disconnected branch of $L+$ solutions (with opposite sense of rotation), which appears above a saddle-node point $N_{d}$. The leading branch of $L-$ solutions and the disconnected branch of $L+$ solutions are eventually stabilized at secondary bifurcation points $S$ and $S_{d}$, respectively, souvenirs of the secondary bifurcation points $S_{1} B_{2}$ found in the horizontal case. When $\theta$ is increased up to $\theta_{c} \approx 0.2714^{\circ}$, the $S_{0}$ and $S$ points on the leading branch will collide and disappear, so that, for larger $\theta$ (up to $\theta=90^{\circ}$ ), the $L-$ solutions on the leading branch become continuously stable in the studied $\mathrm{Ra}$ domain. Another consequence of this collision is that the stable branches of $O \pm$ solutions now belong to disconnected branches above saddle-node points $N \pm$.

In the case of the inclined duct, three types of stable solutions have then been found: the leading longitudinal roll $L-$ solutions, the longitudinal roll $L+$ solutions, and the oblique roll $O \pm$ solutions. We have been able to determine the domains of existence of these different stable solutions in the $\operatorname{Ra}-\theta$ parameter space. The $L-$ solutions are stable at any $\theta$ (up to $\theta=90^{\circ}$ ) and in the $\mathrm{Ra}$ range studied $(\mathrm{Ra} \leqslant 25000)$, except in a small $\mathrm{Ra}$ range $(2726.53<\mathrm{Ra}<$ 3213.62) below $\theta_{c} \approx 0.2714^{\circ}$. The $L+$ solutions are stable beyond the bifurcation point $S_{d}$, i.e., below $\theta \approx 9.445^{\circ}$ and for sufficiently large values of Ra. Finally, the $O \pm$ solutions are stable beyond the secondary bifurcation point $S_{0}$ at very small $\theta$ (below $\theta_{s} \approx 0.263^{\circ}$ ) and then beyond the saddle-node points $N \pm$, i.e., below $\theta \approx 9.235^{\circ}$ and for generally very large values 
of Ra. Five different zones where different stable solutions can coexist have eventually been determined. The possibility or difficulty to reach these stable solutions has been further discussed.

Finally, interesting comparisons with previous studies dealing with convection in inclined ducts have been obtained. These previous studies involved two-dimensional calculations [16], three-dimensional stability studies [19], and experimental measurements [18]. Our results obtained for a tilted finite-length square duct confirm and complement the results obtained in these previous studies. This good agreement suggests that our results are also valid for longer square ducts. The detailed description of the flow behavior obtained thanks to the continuation method is also a motivation for performing new experiments in this domain.

\section{ACKNOWLEDGMENTS}

This work was granted access to the HPC resources of IDRIS under the allocations 2011-021559 and 2012-021559 made by GENCI (Grand Equipement National de Calcul Intensif). We would like to extend our utmost gratitude to the Tohoku University Global COE program for the financial support, as well as to the administration of Ecole Centrale de Lyon and Tohoku University for making possible the collaboration on the joint double-degree Ph.D. program.
[1] H. Bénard, Ann. Chim. Phys. 23, 62 (1901).

[2] Lord Rayleigh, Philos. Mag. 32, 529 (1916).

[3] M. Lappa, C. R. Mecanique 335, 253 (2007).

[4] J. R. Baumgardner, J. Stat. Phys. 39, 501 (1985).

[5] M. Meneguzzi, C. Sulem, P. L. Sulem, and O. Thual, J. Fluid Mech. 182, 169 (1987).

[6] E. Bodenschatz, W. Pesch, and G. Ahlers, Annu. Rev. Fluid Mech. 32, 709 (2000).

[7] K. A. Cliffe and K. H. Winters, J. Comput. Phys. 67, 310 (1986).

[8] I. Catton, ASME J. Heat Transfer 92, 186 (1970).

[9] G. S. Charlson and R. L. Sani, Int. J. Heat Mass Transfer 13, 1479 (1970).

[10] R. Touihri, A. El Gallaf, D. Henry, and H. Ben Hadid, Phys. Rev. E 84, 056302 (2011).

[11] D. Puigjaner, J. Herrero, F. Giralt, and C. Simó, Phys. Fluids 16, 3639 (2004).

[12] D. Puigjaner, J. Herrero, F. Giralt, and C. Simó, Phys. Rev. E 73, 046304 (2006).

[13] D. Puigjaner, J. Herrero, C. Simó, and F. Giralt, J. Fluid Mech. 598, 393 (2008).

[14] J. Pallares, M. P. Arroyo, F. X. Grau, and F. Giralt, Exp. Fluids 31, 208 (2001).

[15] J. Pallares, F. X. Grau, and F. Giralt, Int. J. Heat Mass Transfer 42, 753 (1999).
[16] K. A. Cliffe and K. H. Winters, J. Comput. Phys. 54, 531 (1984).

[17] K. R. Kirchartz and H. Oertel, Jr., J. Fluid Mech. 192, 249 (1988).

[18] H. Ozoe, H. Sayama, and S. W. Churchill, Int. J. Heat Mass Transfer 17, 401 (1974).

[19] T. Adachi, Int. J. Heat Mass Transfer 49, 2372 (2006).

[20] H. Ben Hadid and D. Henry, J. Fluid Mech. 333, 57 (1997).

[21] G. E. Karniadakis, M. Israeli, and S. A. Orszag, J. Comput. Phys. 97, 414 (1991).

[22] C. K. Mamun and L. S. Tuckerman, Phys. Fluids 7, 80 (1995).

[23] A. Bergeon, D. Henry, H. Ben Hadid, and L. S. Tuckerman, J. Fluid Mech. 375, 143 (1998).

[24] G. Petrone, E. Chénier, and G. Lauriat, Int. J. Heat Mass Transfer 47, 3889 (2004).

[25] T. C. Oppe, W. D. Joubert, and D. R. Kincaid, Comput. Phys. Commun. 53, 283 (1989).

[26] R. B. Lehoucq, D. C. Sorensen, and C. Yang, ARPACK Users' Guide: Solution of Large-scale Eigenvalue Problems with Implicitly Restarted Arnoldi Methods (SIAM, Philadelphia, 1998).

[27] S. Wakashima and T. S. Saitoh, Int. J. Heat Mass Transfer 47, 853 (2004). 